Prepared for the U.S. Department of Energy

Under Contract DE-AC05-76RL01830

\title{
FY08 Annual Report for Nuclear Resonance Fluorescence Imaging
}

\author{
GA Warren \\ JA Caggiano
}

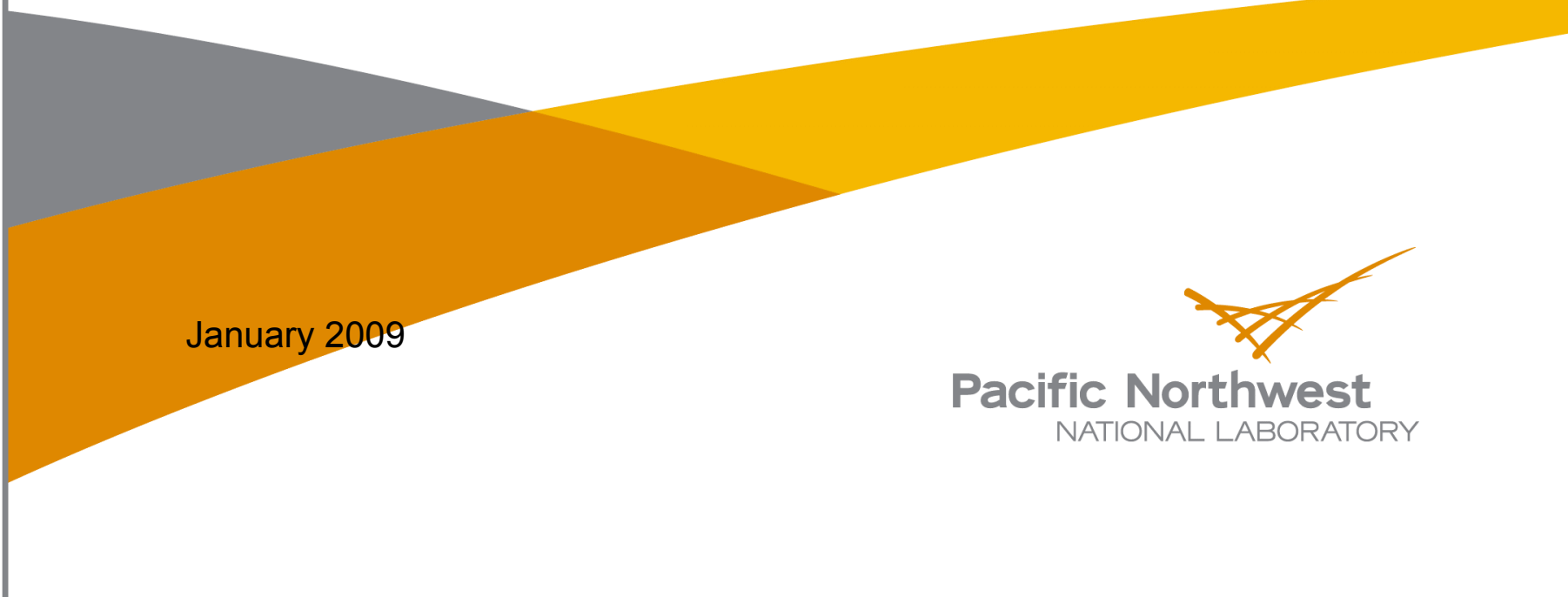




\title{
DISCLAIMER
}

This report was prepared as an account of work sponsored by an agency of the United States Government. Neither the United States Government nor any agency thereof, nor Battelle Memorial Institute, nor any of their employees, makes any warranty, express or implied, or assumes any legal liability or responsibility for the accuracy, completeness, or usefulness of any information, apparatus, product, or process disclosed, or represents that its use would not infringe privately owned rights. Reference herein to any specific commercial product, process, or service by trade name, trademark, manufacturer, or otherwise does not necessarily constitute or imply its endorsement, recommendation, or favoring by the United States Government or any agency thereof, or Battelle Memorial Institute. The views and opinions of authors expressed herein do not necessarily state or reflect those of the United States Government or any agency thereof.

\author{
PACIFIC NORTHWEST NATIONAL LABORATORY \\ operated by \\ BATTELLE \\ for the \\ UNITED STATES DEPARTMENT OF ENERGY \\ under Contract DE-AC05-76RL01830
}

Printed in the United States of America
Available to DOE and DOE contractors from the Office of Scientific and Technical Information,
P.O. Box 62, Oak Ridge, TN 37831-0062;
ph: (865) 576-8401
fax: $(865) 576-5728$
email: reports@adonis.osti.gov

\footnotetext{
Available to the public from the National Technical Information Service, U.S. Department of Commerce, 5285 Port Royal Rd., Springfield, VA 22161 ph: (800) 553-6847 fax: $(703) 605-6900$ email: orders@ntis.fedworld.gov online ordering: http://www.ntis.gov/ordering.htm
}

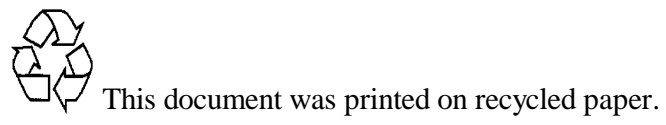




\title{
FY08 Annual Report for Nuclear Resonance Fluorescence Imaging
}

\author{
GA Warren \\ JA Caggiano
}

January 2009

Prepared for

the U.S. Department of Energy

under Contract DE-AC05-76RL01830

Pacific Northwest National Laboratory

Richland, Washington 99352 



\section{Executive Summary}

The overall objective of this project was to investigate the potential of nuclear resonance fluorescence (NRF) to address national security applications. NRF is a physics process that provides isotopic specific signatures using photon-beams. This report describes both the results of the FY08 effort, as well as a summary of the important conclusions from previous years of the project.

The important findings of this project are as follows:

- ${ }^{235} \mathrm{U}$ has strong NRF signatures between 1650 and $2050 \mathrm{keV}$. The strongest signature is at $1733 \mathrm{keV}$ with an integrated cross section of $30 \mathrm{eV} \mathrm{b}$, which is large for an NRF signature.

- No resonances in ${ }^{235} \mathrm{U}$ above roughly $5 \mathrm{eV}$ b in the 2050 to $5000 \mathrm{keV}$ range were observed.

- NRF measurements conducted with a narrow bandwidth photon source have several distinct advantages over measurements conducted with a bremsstrahlung photon source. One advantage is the higher signal-to-noise ratio of the observed peaks due to the narrow energy distribution of incident photons. Another advantage for laboratory measurements is higher signal rate due to the reduced background and smaller target-to-detector distances made possible by the well-defined photon beam.

- The CW bremsstrahlung source, compared to the pulsed bremsstrahlung source and narrow bandwidth source, provided the highest signal rate when the measurement rates were normalized to compare the same geometries.

- NRF measurements conducted with a pulsed bremsstrahlung source is limited to a detector rate of approximately one-tenth of the repetition rate of the beam to minimize the effects of pileup in the detector. Thus, for a $1 \mathrm{kHz}$ machine, the detector rate is limited to about $100 \mathrm{~Hz}$. This detector rate is significantly less than $10-15 \mathrm{kHz}$ rate commonly achieved for NRF measurements using a CW bremsstrahlung source.

- The signal-to-noise ratio can be very sensitive to the probability for pileup. For the $4842-\mathrm{keV}$ line of lead, a $5 \%$ probability of pileup reduced the signal-to-noise by a factor of 2 . This effect is critical for pulsed bremsstrahlung measurements where pileup is more likely. 



\section{Acronyms and Abbreviations}

CW: Continuous Wave

DU: Depleted uranium

HEU: Highly enriched uranium

HPGe: high purity germanium

IAC: Idaho Accelerator Center

MDIC: Minimum Detectable Integrated Cross Section

MIT: Massachusetts Institute of Technology

NRF: Nuclear Resonance Fluorescence

S/N: Signal-to-Noise Ratio

TUD: Technical University Darmstadt

UCSB: University California Santa Barbara 



\section{Contents}

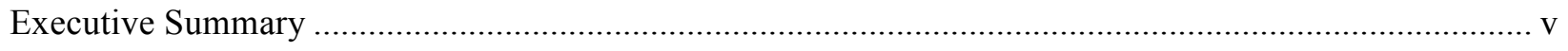

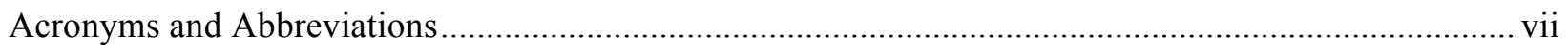

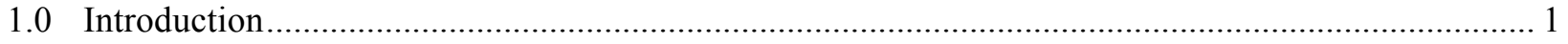

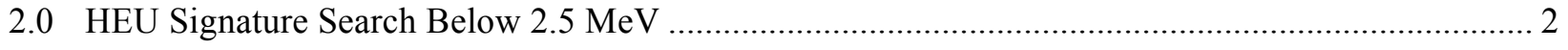

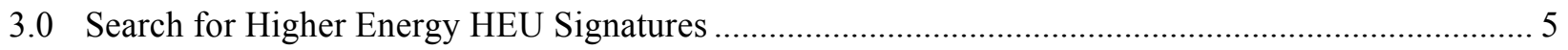

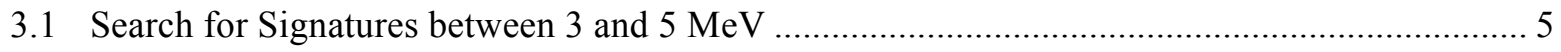

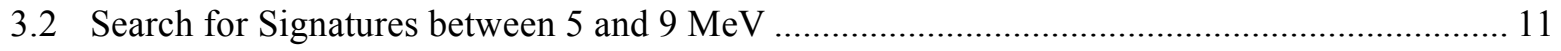

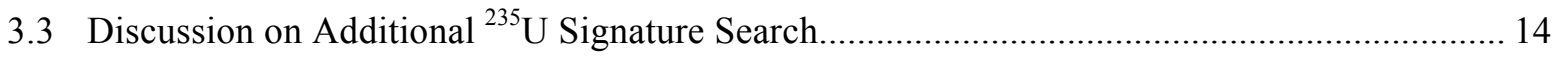

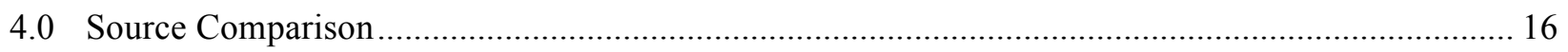

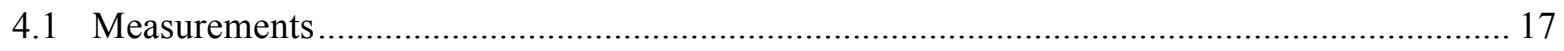

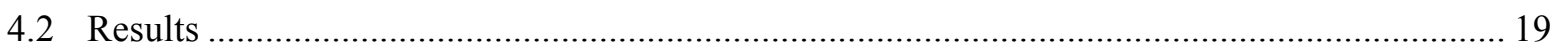

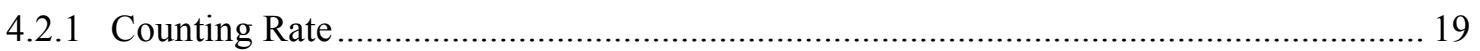

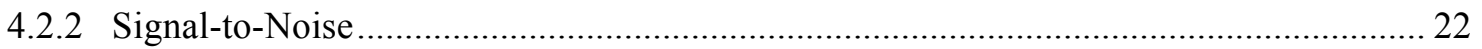

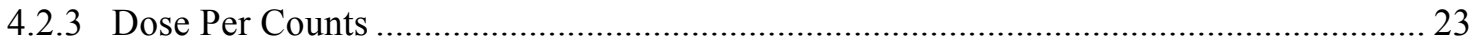

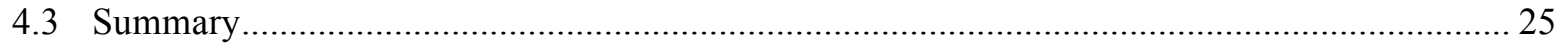

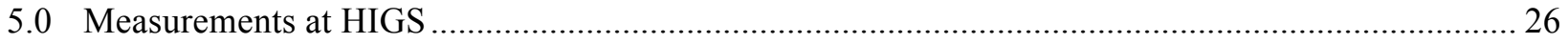

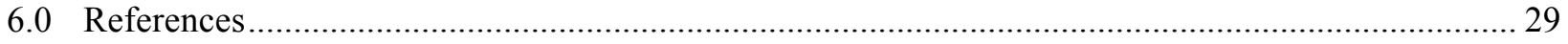




\section{Figures}

Figure 2.1. X-ray image of the HEU sample used to measure the ${ }^{235} \mathrm{U}$ NRF cross sections. ...................... 2

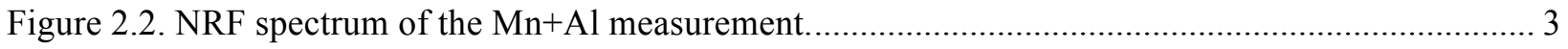

Figure 2.3. NRF Spectrum from measurement on HEU+Mn. ...................................................... 4

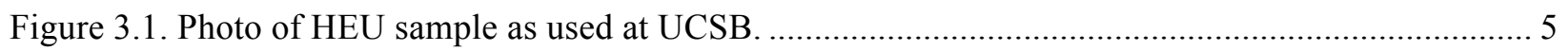

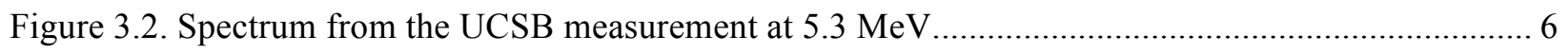

Figure 3.3. Low energy portion of the spectrum from the UCSB measurements................................... 7

Figure 3.4. High energy portion of the spectrum from the UCSB HEU measurements............................ 7

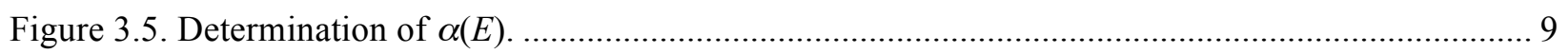

Figure 3.6. Minimum detectable integrated cross section from the UCSB measurements........................ 9

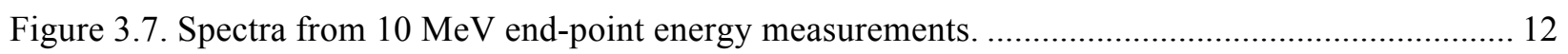

Figure 3.8. Expanded view of two peaks of unknown origin, left is at $8210 \mathrm{keV}$ and right is at

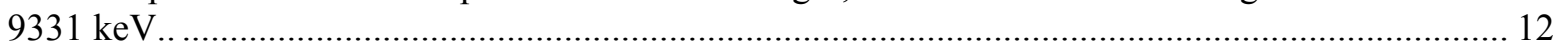

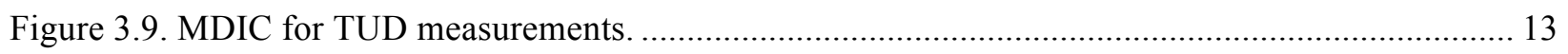

Figure 4.1. Comparison of energy distribution of $5.3 \mathrm{MeV}$ end-point energy bremsstrahlung distribution and 3\% FWHM narrow bandwidth distribution centered at $4.8 \mathrm{MeV}$. ......................... 16

Figure 4.2. Comparison of NRF spectra on 1/8" lead using three different photon sources. .................... 20

Figure 4.3. Counts to detection as a function of the $\mathrm{S} / \mathrm{N}$ ratio for $A_{P D}=1.6$ and $A_{F A}=3.1 \ldots \ldots \ldots \ldots \ldots \ldots \ldots . .24$

Figure 5.1. NRF spectra of DU from a CW bremsstrahlung source and a narrow bandwidth

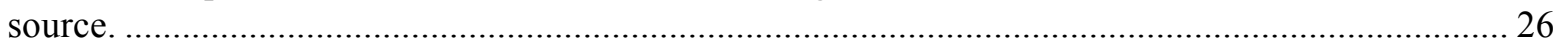

Figure 5.2. Spectra from Z-study of photon scattering. See text for details. ........................................ 27 


\section{Tables}

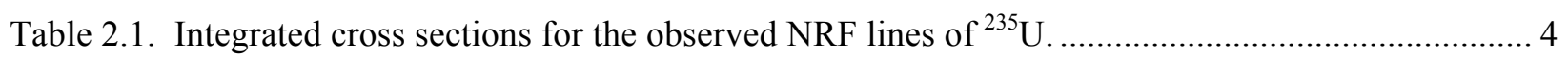

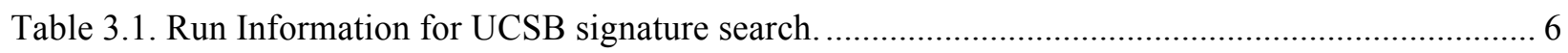

Table 3.2. Amount of shielding material necssary to compensate for the minimum factor of 11 difference in count rate between the known $1733 \mathrm{keV}$ resonance and a hypothetical

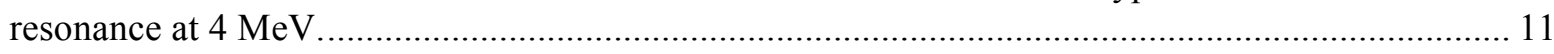

Table 4.1. Properties of photon source comparison measurements. ...................................................... 18

Table 4.2. Summary of count rate results for different photon sources. See text for details.................... 21

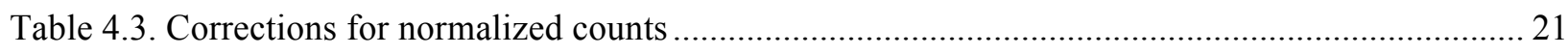

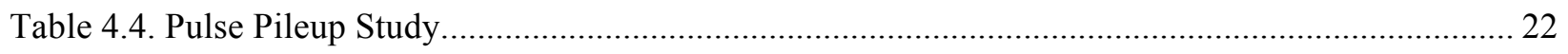

Table 5.1. Comparison of Compton peak and 511-keV peak strengths in the Z-study for photon

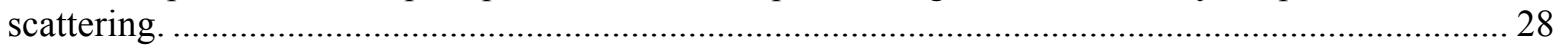





\subsection{Introduction}

Nuclear resonance fluorescence (NRF) is a well-established physical process [Metzger-59][Kniessl-96] that provides an isotope-specific signature that can be exploited for isotopic detection and characterization of samples [Bertozzi-05]. The physical process behind NRF is the nuclear absorption of photons at discrete energy levels followed by the electromagnetic de-excitation of the excited nucleus. Through that process, one or more photons will be emitted roughly isotropically (typically a dipole angular distribution). The energies of the emitted photons are characteristic of the individual isotope of the element. This unique information provides a means to identify specific isotopes, either stable or radioactive.

One isotope of major interest for possible NRF applications is ${ }^{235} \mathrm{U}$. Prior to this project, the understanding of the nuclear levels of ${ }^{235} \mathrm{U}$ did not include any states that would have an obvious NRF response [Firestone-99]. This project conducted signature searches of ${ }^{235} \mathrm{U}$ in two phases. In the first phase, measurements were conducted at the Massachusetts Institute of Technology (MIT) to search for signatures below $2.5 \mathrm{MeV}$. In the second phase, measurements were conducted at the University of California Santa Barbara (UCSB) to search for signatures between 2.5 and $5.0 \mathrm{MeV}$. While not explicitly a part of this project, a review of the signature search on HEU and DU above $5 \mathrm{MeV}$ is included for completeness.

When developing potential applications of NRF one must determine the optimal photon source for that application. There are a variety of ways to create photons suitable for conducting NRF measurements. To assist in that evaluation, PNNL conducted similar measurements on a lead foil using three different sources: a $100 \%$ duty factor bremsstrahlung source, a narrow bandwidth source and a pulsed bremsstrahlung source. Issues such a measured count rate, count rate normalized to the same geometry, signal-to-noise and dose are examined. In addition to the lead measurements, measurements on depleted uranium (DU) using a high-duty factor bremsstrahlung source and the narrow bandwidth photon source are compared.

This report is written as a review for the entire project. As such, it begins with a brief review of the important findings of the first two years, which focused on NRF signature search of ${ }^{235} \mathrm{U}$. The report concludes with a comparison of the performance of different photon sources. 


\subsection{HEU Signature Search Below 2.5 MeV}

In August 2006, PNNL in collaboration with Passport Systems conducted a series of measurements to determine the NRF cross sections of ${ }^{235} \mathrm{U}$. A collection of NRF signatures was observed between 1650 and $2050 \mathrm{keV}$. Analysis of these results were refined over the subsequent months, reducing the (1-sigma) uncertainty from the initial estimated $30 \%$ down to $13 \%$ for the strongest signature. The principle refinements were improved understanding of the impact of the target geometry on the measurement and more reliable cross sections for the normalization material.

The HEU target used to perform the measurements of the ${ }^{235} U$ cross sections was composed of several foils stacked into a $\sim 3.5$ " diameter plastic container. The foils were arranged carefully to keep the thickness as uniform as possible. However, variations of the thickness across the sample remained. In the preliminary analysis of the ${ }^{235} \mathrm{U}$ cross sections, the thickness of the HEU was not well known so that it was conservatively estimated that the thickness varied from 1.9 to $5.7 \mathrm{~mm}$ for a constant mass of HEU. This uncertainty in the thickness of the sample introduces an uncertainty of $30 \%$ to the measured cross section.

The composite HEU target used in the MIT measurements was x-rayed to determine its variation in thickness (see Figure 2.1), and thus its contribution to the uncertainty in the measured ${ }^{235} \mathrm{U}$ NRF cross sections. Analyzing the x-ray images of the sample demonstrated that the thickness of the sample varied much less than the original estimate; it was determined that the sample thickness was $1.9 \pm 0.5 \mathrm{~mm}$.

The uncertainty in the cross section due to uncertainty in the thickness depends on the absorption of the incoming bremsstrahlung photons and outgoing, fluorescent photons by the HEU. This absorption

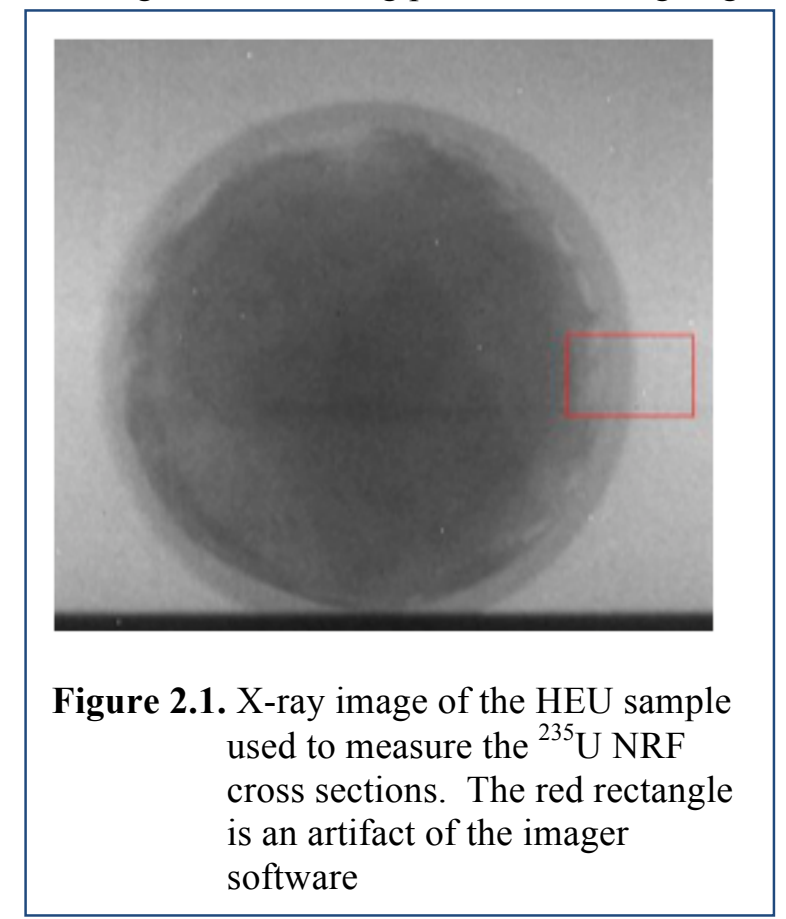
depends on the thickness of the HEU. The fixed mass of HEU is an important constraint. The profile of the thicknesses, i.e. the number of pixels for each bin of thickness, for the four different radiography analyses was used to determine the average absorption. The four analyses differed in the functional form of the background, linear or quadratic, for two different radiographic images. The uncertainty in cross section due to different absorptions from those four profiles was only $0.5 \%$. To allow for other possible model-dependent uncertainties, the final uncertainty was determined from the variation in the absorption for two disks of HEU, both of the same mass and density but 0.95 and $2.85 \mathrm{~mm}$ in thickness. The absorption for these two extreme cases varied by $1.5 \%$, which is the uncertainty attributed to the cross sections of the ${ }^{235} \mathrm{U}$ NRF lines due to variations in the target thickness.

The ${ }^{235} \mathrm{U}$ NRF cross sections were measured relative to the NRF cross sections of the $1884-\mathrm{keV}$ state in ${ }^{55} \mathrm{Mn}$, and is therefore reliant on an accurate ${ }^{55} \mathrm{Mn}$ cross section. Preliminary analysis suggested the ${ }^{55} \mathrm{Mn}(1884) \mathrm{NRF}$ cross section differed from the literature value by $\sim 30 \%$. Deeper investigation into the evaluated width of the 1884-keV state in ENSDF [Tuli-87] revealed that its evaluated half-life of $T_{1 / 2}=$ $12 \pm 3$ fs was based on three measurements: $9 \pm 2$ fs from evaluated ${ }^{55} \operatorname{Mn}\left(\left(\mathrm{n}, \mathrm{n}^{\prime} \gamma\right), 19 \pm 3\right.$ fs from evaluated ${ }^{54} \operatorname{Cr}(\mathrm{p}, \gamma)$ and $11 \pm 8 \mathrm{fs}$ from evaluated ${ }^{52} \operatorname{Cr}(\langle, \mathrm{p} \gamma)$. In addition, our preliminary analysis of the 


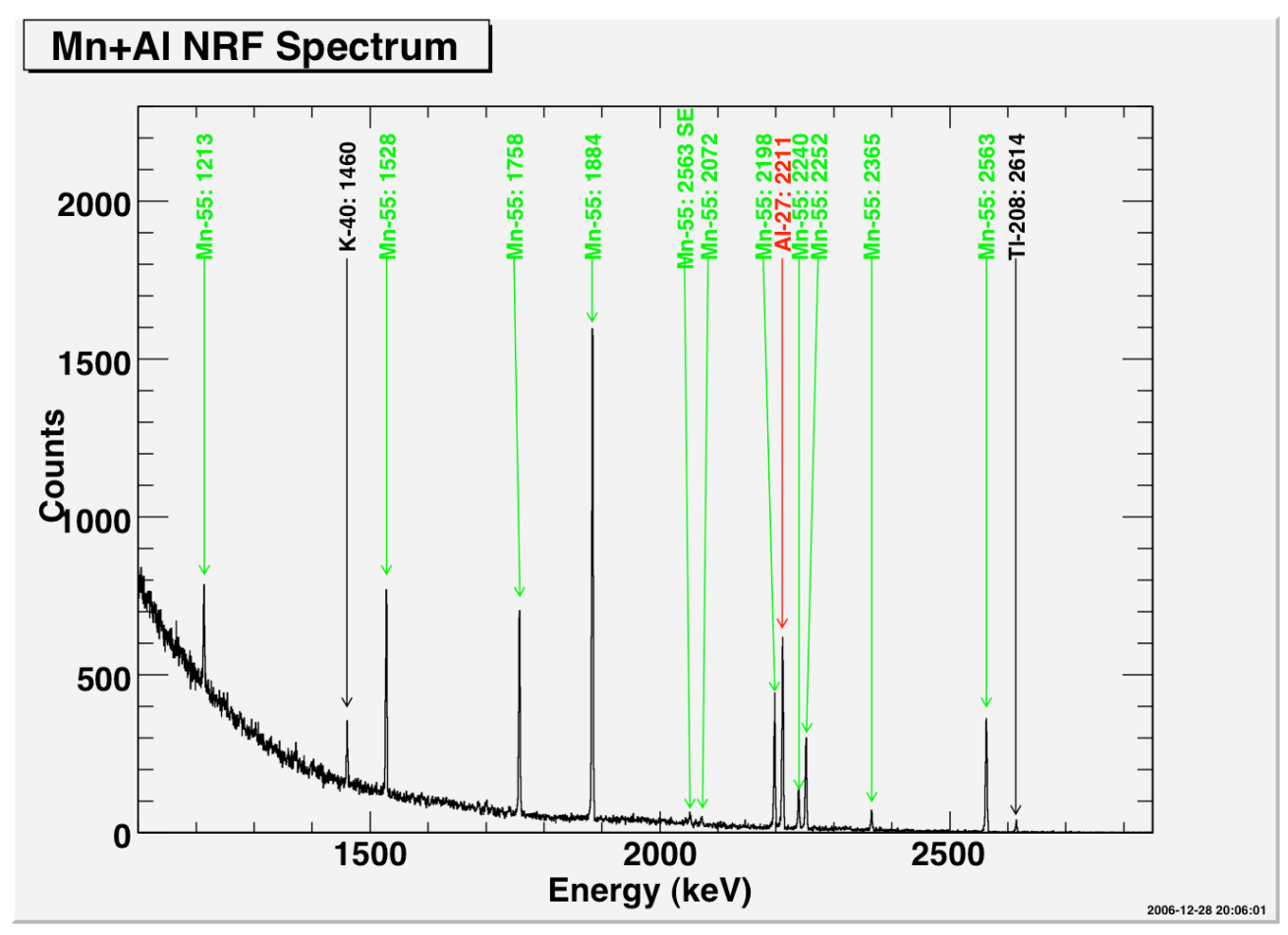

Figure 2.2. NRF spectrum of the $\mathrm{Mn}+\mathrm{Al}$ measurement.

width of the 1884-keV state based on NRF measurements agreed with previous NRF measurements [Alston-68], once the previous methods were corrected for the evaluated branching ratio of the $1884 \mathrm{keV}$ state to the ground state. Therefore, it was determined that the higher accuracy measurements of the ${ }^{55} \mathrm{Mn}(1884)$ line were necessary.

The ${ }^{55} \mathrm{Mn}(1884) \mathrm{NRF}$ cross section was measured relative to the well-known NRF line in aluminum at $2212 \mathrm{keV}$. The measurements were conducted by Passport at the MIT High-voltage Research Laboratory (HVRL) with a $2.8 \mathrm{MeV}$ bremsstrahlung photon beam. The targets were $\sim 220$ gram discs of manganese and aluminum. Two measurements were performed, one with the manganese upstream of the aluminum and the other vice versa. Figure 2.2 shows the HPGe (100\% relative efficiency) spectrum taken with the manganese target upstream of the aluminum target. The measured width for the ${ }^{55} \mathrm{Mn}(1884) \mathrm{keV}$ state was determined to be $7.66 \pm 0.63 \mathrm{fs}$. This result is consistent with two out of the three measurements used to determine the evaluated width in ENSDF and with the Alston et al. measurement [Alston-68]. This measurement uncertainty of the ${ }^{55} \mathrm{Mn}(1884)$ width translates into a $12 \%$ uncertainty in the resonances of ${ }^{235} \mathrm{U}$, which is the largest contribution to the uncertainty of the strongest ${ }^{235} \mathrm{U}$ line at 1733 $\mathrm{keV}$.

The final analysis for the integrated cross sections for the ${ }^{235} \mathrm{U}$ NRF lines is shown in Table 2.1, while the spectrum is shown in Figure 2.3. Only those peaks with a significance greater than 5 are shown; the significance is defined as the counts in the peak divided by the statistical and systematic uncertainties added in quadrature. Of these nine peaks, the line at $1733.6 \mathrm{keV}$ is the strongest at $30 \mathrm{eV} \mathrm{b}$, which is large for an NRF cross section. Another interesting observation is the pair of the lines at 1687.3 and $1733.6 \mathrm{keV}$ and 1769.2 and $1815.3 \mathrm{keV}$. Both of these pairs of lines are separated by $46.2 \mathrm{keV}$ (within errors), suggesting that the state is decaying to the ground state and the $9 / 2$ - state of ${ }^{235} \mathrm{U}$ at $46.2 \mathrm{keV}$. This pairing of states provides strong circumstantial evidence that the observed resonances are due to ${ }^{235} \mathrm{U}$. The results of the ${ }^{235} \mathrm{U}$ measurements were recently published as [Bertozzi-08]. 


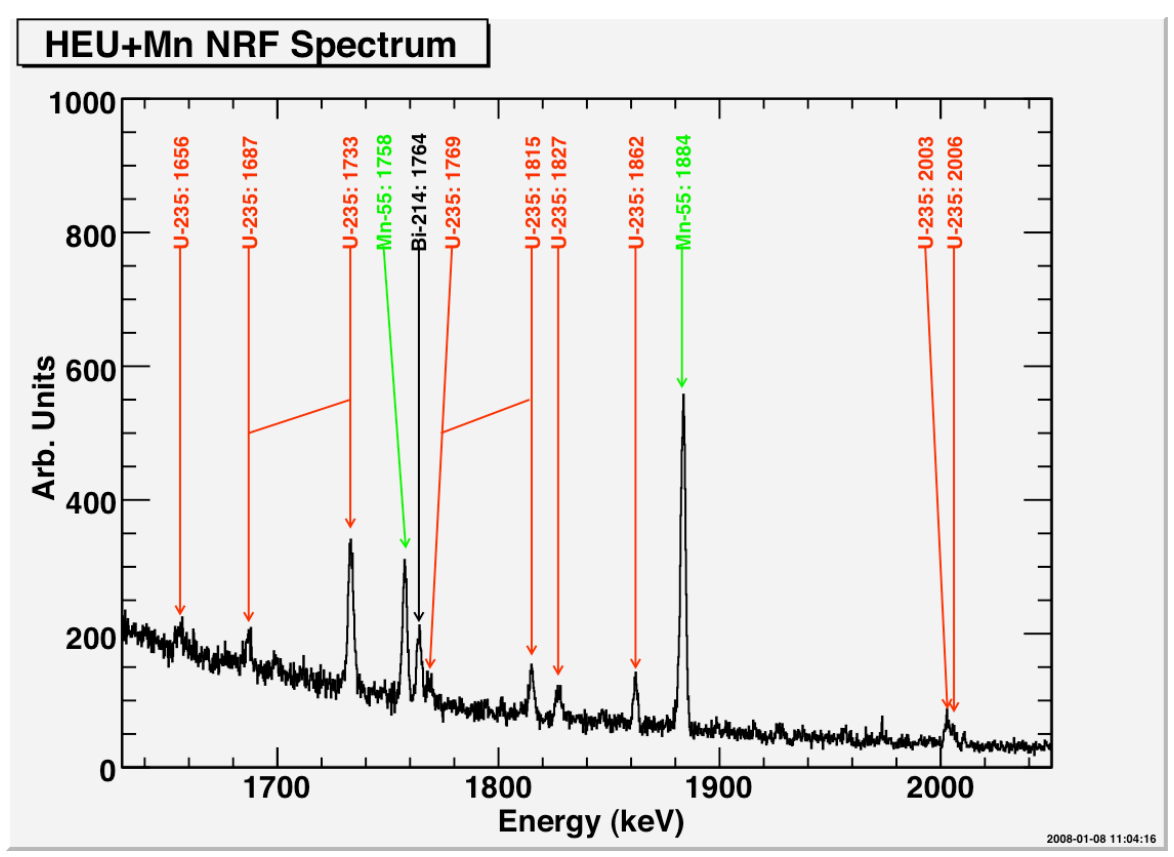

Figure 2.3. NRF Spectrum from measurement on $\mathrm{HEU}+\mathrm{Mn}$.

Table 2.1. Integrated cross sections for the observed NRF lines of ${ }^{235} \mathrm{U}$.

\begin{tabular}{|c|r|}
\hline $\begin{array}{c}\text { Energy } \\
(\mathrm{keV})\end{array}$ & $\begin{array}{c}\text { Int. Cross Section } \\
(\mathrm{eV} \mathrm{b})\end{array}$ \\
\hline $1656.23 \pm 0.80$ & $4.1 \pm 1.3$ \\
\hline $1687.26 \pm 0.33$ & $6.1 \pm 1.1$ \\
\hline $1733.60 \pm 0.22$ & $29.8 \pm 3.9$ \\
\hline $1769.16 \pm 0.28$ & $4.4 \pm 1.0$ \\
\hline $1815.31 \pm 0.22$ & $9.7 \pm 1.7$ \\
\hline $1827.54 \pm 0.23$ & $6.7 \pm 1.2$ \\
\hline $1862.31 \pm 0.20$ & $9.6 \pm 1.7$ \\
\hline $2003.32 \pm 0.25$ & $9.7 \pm 1.7$ \\
\hline $2006.19 \pm 0.31$ & $4.7 \pm 1.6$ \\
\hline
\end{tabular}




\subsection{Search for Higher Energy HEU Signatures}

\subsection{Search for Signatures between 3 and $5 \mathrm{MeV}$}

The measurements discussed in the previous section were limited to signature energies below about $2.8 \mathrm{MeV}$ because of the maximum energy of the MIT accelerator. Additional ${ }^{235} \mathrm{U}$ NRF signatures search measurements were conducted in June and July of 2007 to search for new NRF lines from 3 to $5 \mathrm{MeV}$. These higher energy measurements were conducted in collaboration with Passport at the University of California Santa Barbara (UCSB) Free Electron Laser facility.

A new HEU sample was necessary to conduct measurements at UCSB. The NRC license for UCSB limited their ${ }^{235} \mathrm{U}$ inventory to $10 \mathrm{~g}$, so the $220 \mathrm{~g}$ sample used for the MIT measurements could not be used. The HEU for the new sample was taken from existing inventory. The sample consisted of 23 rectangular foils approximately $1.5 \mathrm{~cm} \times 1.0 \mathrm{~cm}$. The $93 \%$ enriched HEU weighed a total of $7.7 \mathrm{~g}$. The HEU was packed into four separate polycarbonate containers. A photo of the sample, as used at UCSB, is shown in Figure 3.1.

The HEU was arranged in four separate containers so it would be easy to reconfigure the sample for

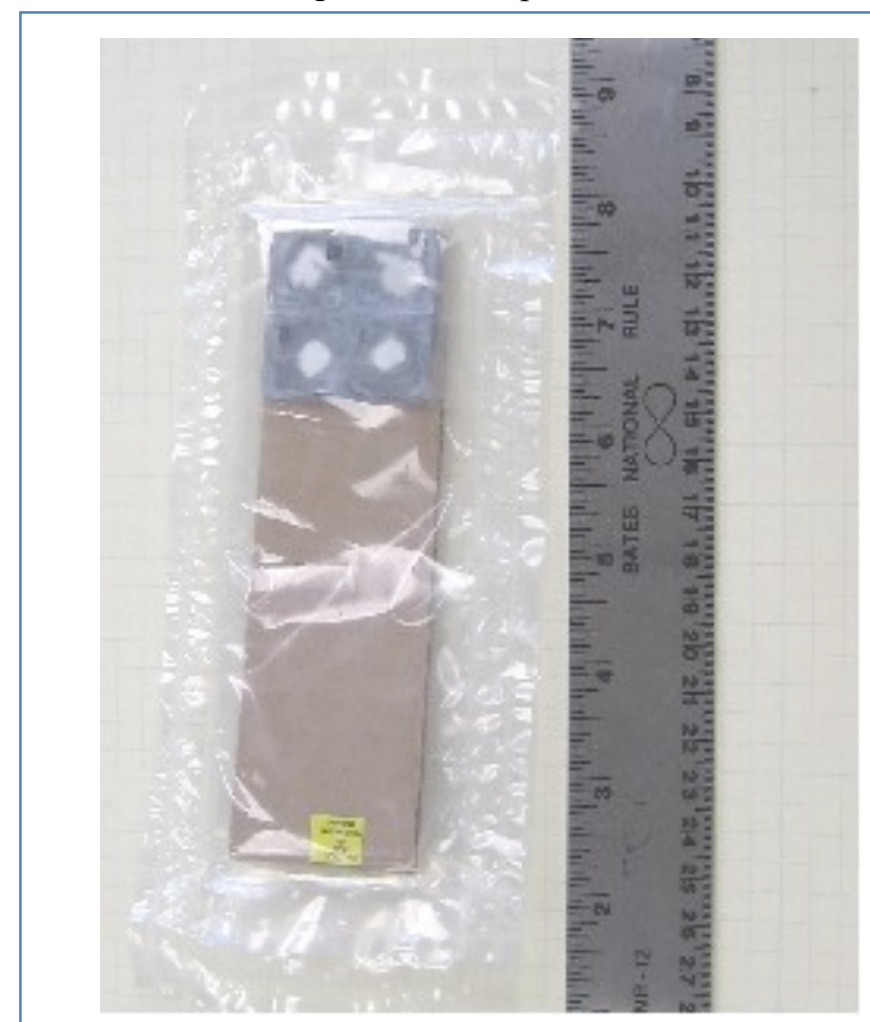

Figure 3.1. Photo of HEU sample as used at UCSB. The long sheet of cardboard enabled easy mounting for the measurement. The round white material in the polycarbonate squares was cotton placed to keep the HEU from shifting inside the containers. measurements conducted at Technical University Darmstadt (TUD) during August 2007. Those measurements were funded by DNDO and will be briefly discussed in Section 3.2. The TUD beam is considerably smaller in diameter, so the containers were stacked on top of each other to minimize the cross sectional area presented to the beam.

The measurements were conducted in June and July 2007 at the UCSB Free Electron Laser Facility. The 100\% duty factor electron beam was incident upon a tantalum plated copper radiator to generate the bremsstrahlung photon beam. The beam was then collimated to form a cone of photons directed at the target. The entire target was illuminated by the photon beam. Three HPGe detectors were used to detector the scattered photons. The detectors were shielded by 8 " of lead on all sides except for the side facing the target, which had a 1" lead filter to reduce the flux from low energy photons. Each detector had a relative efficiency of about $100 \%$. Measurements were also conducted on empty containers to confirm that any new peaks cannot be attributed to the container itself, rather than the HEU. 
Table 3.1. Run Information for UCSB signature search.

\begin{tabular}{|l|c|c|c|c|c|}
\hline \multicolumn{1}{|c|}{ Target } & $\begin{array}{c}\text { Beam } \\
\text { Energy } \\
(\mathrm{MeV})\end{array}$ & $\begin{array}{c}<\mathrm{I}> \\
(\mu \mathrm{A})\end{array}$ & Charge & $\begin{array}{c}\text { Real } \\
\text { Time } \\
(\mathrm{C})\end{array}$ & $\begin{array}{c}\text { Dead } \\
\text { Time } \\
(\%)\end{array}$ \\
\hline HEU & 4.3 & 116.2 & 7.9 & 18.2 & 16 \\
\hline HEU + Al & 5.1 & 59.2 & 4.7 & 22.1 & 19 \\
\hline HEU & 5.3 & 49.7 & 6.9 & 38.5 & 18 \\
\hline
\end{tabular}

Measurements were conducted a beam energies of 4.3, 5.1 and $5.3 \mathrm{MeV}$. The first measurement was conducted at $5.1 \mathrm{MeV}$. In addition to the HEU sample, a $4 \mathrm{~g}$ aluminum plate was inserted into the beam. As will be discussed in more detail later, the NRF lines from aluminum provided beam flux normalization. After conditioning the accelerator at $5.1 \mathrm{MeV}$, the beam energy was raised to $5.3 \mathrm{MeV}$ to increase the photon flux at the maximum energies. Measurements were also conducted at $4.3 \mathrm{MeV}$ to provide more sensitivity to signatures at lower energies. A summary of the run information for the UCSB runs is provided in Table 3.1.

The observed spectra from these measurements, which are shown in Figure 3.2 through Figure 3.4, have several interesting features:

- There were no significant peaks above $2.1 \mathrm{MeV}$ that could be attributed to ${ }^{235} \mathrm{U}$. We defined "significant" as a peak area greater than 5 times the uncertainty of that peak. The peak uncertainty was determined by adding, in quadrature uncertainties, from the peak and from the background.

\section{Full NRF Spectra}

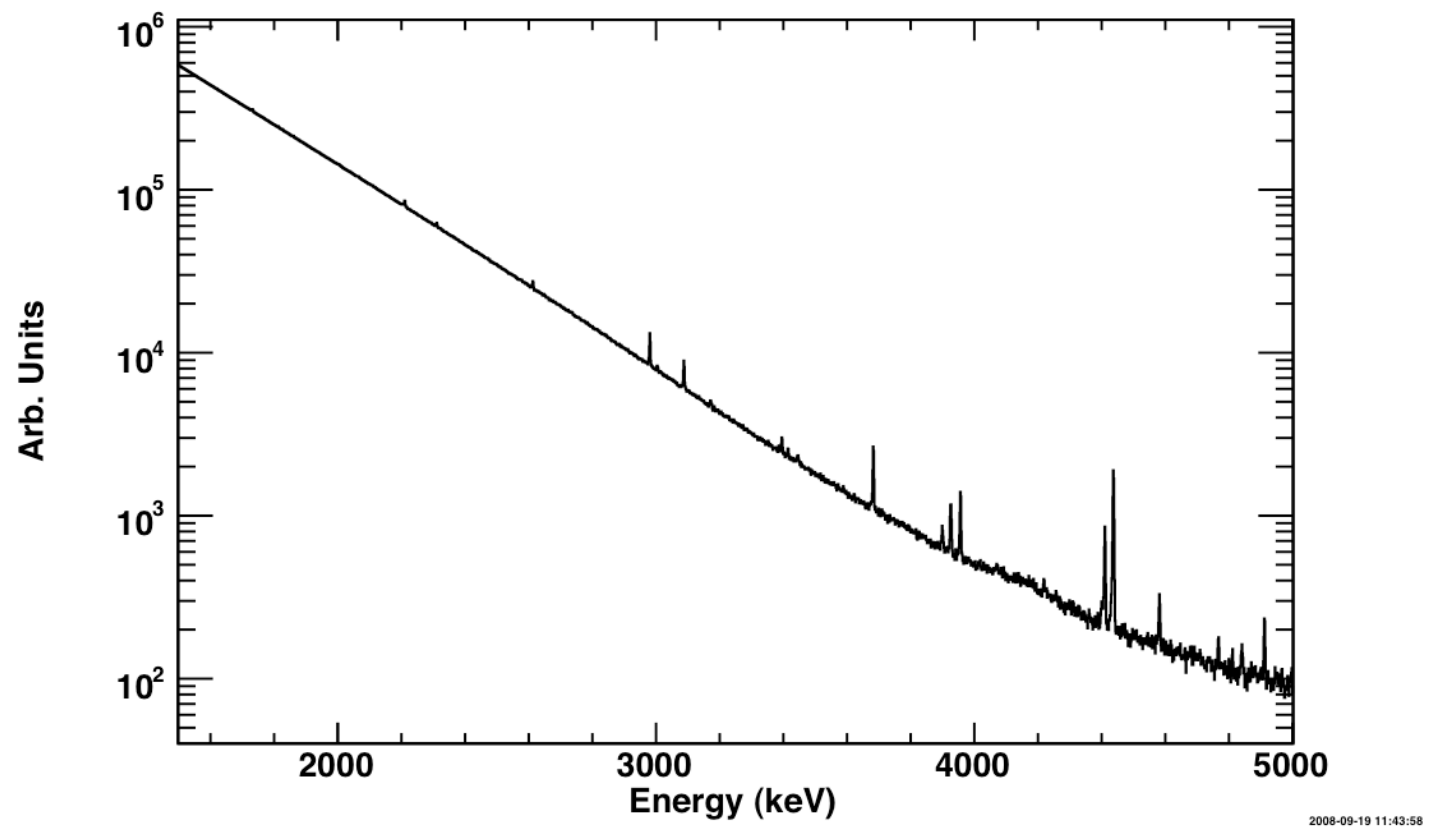

Figure 3.2. Spectrum from the UCSB measurement at $5.3 \mathrm{MeV}$. 


\section{Continuum Subtracted NRF Spectra}

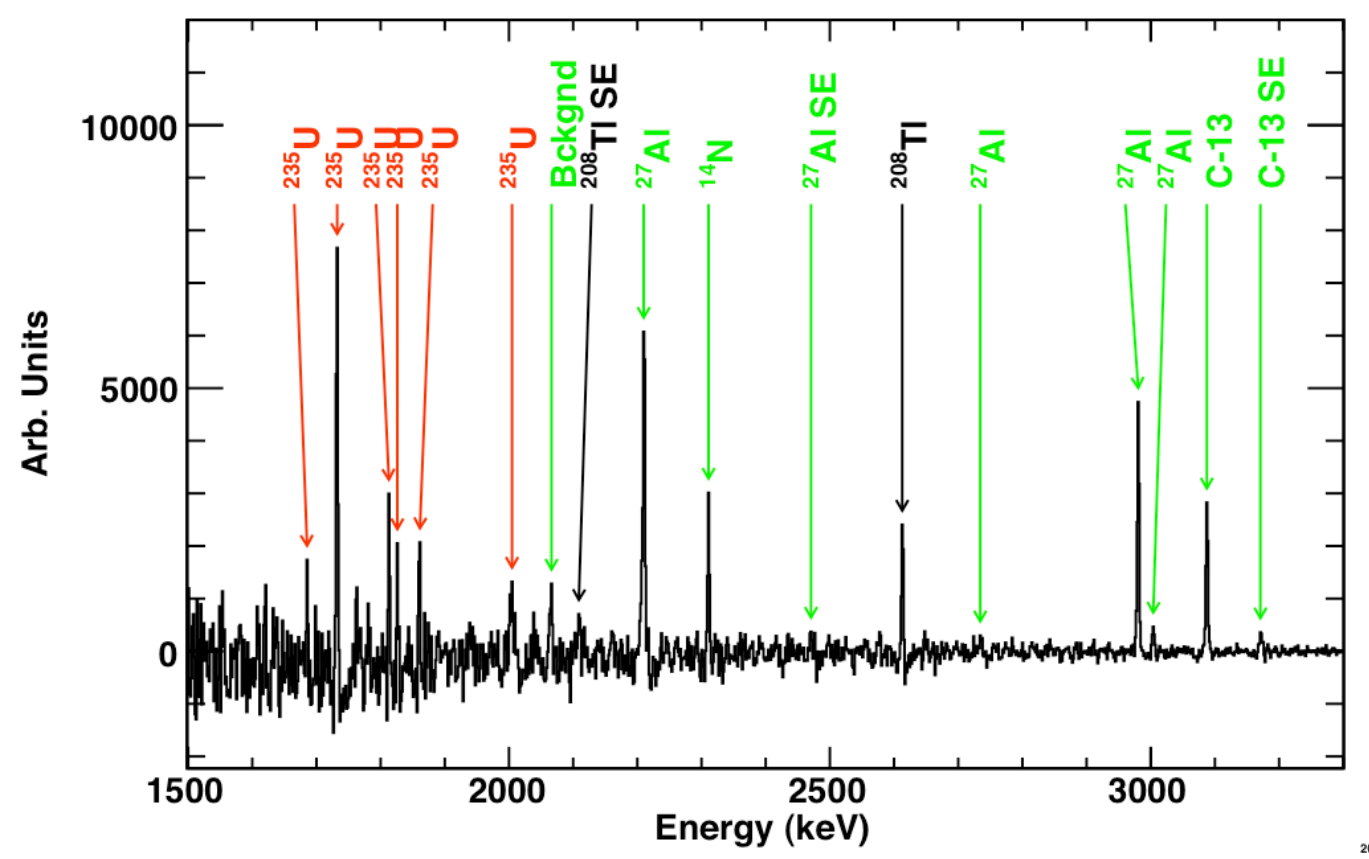

Figure 3.3. Low energy portion of the spectrum from the UCSB measurements. The background continuum has been subtracted.

\section{Continuum Subtracted NRF Spectra}

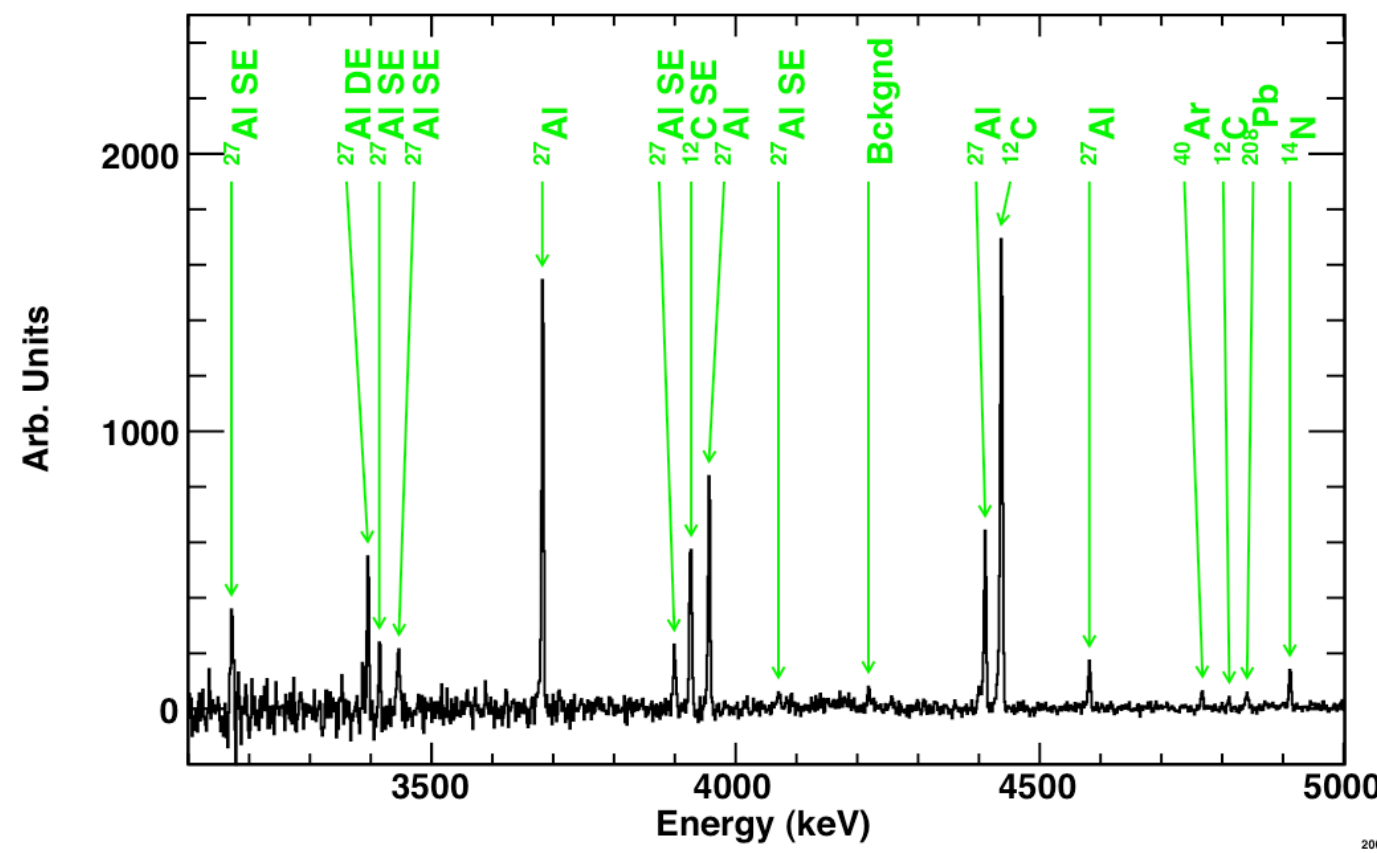

Figure 3.4. High energy portion of the spectrum from the UCSB HEU measurements. The background continuum has been subtracted. 
- The $1733-\mathrm{keV}$ line of ${ }^{235} \mathrm{U}$ observed previously is clearly visible in the $5.3 \mathrm{MeV}$ data. This observation is significant for two reasons: 1) It confirms the earlier observation as this measurement was conducted using an entirely different experimental setup (different photon source, different HEU, different detectors) and 2) It is impressive that this state can still be observed so far below the end-point energy of the photon distribution considering the increased background.

- There are significant peaks from both argon and nitrogen. These peaks originate from the air around the target. The observation of the argon peak, even though argon is only $0.9 \%$ of the atmosphere, is made possible by the unusually large NRF cross section $(\sim 400 \mathrm{eV} \mathrm{b})$.

- There are two peaks labeled as "Background." These peaks appear in both the spectra with and without the HEU, but not in the beam-off background measurements, so they are likely attributed to an isotope in the container.

As no new NRF response of ${ }^{235} \mathrm{U}$ was observed, it is important to determine the maximum cross section that could have gone unobserved in these measurements. We refer to this sensitivity threshold as the minimum detectable integrated cross section (MDIC). A potential peak would be declared as significant if the counts in the peak were more than 5 times the statistical uncertainty of the peak ${ }^{1}$. That statistical uncertainty includes both the uncertainty of the peak and the uncertainty of the background added in quadrature. As a result, if there were $B$ counts in the background, the peak counts would need to be at least

$$
P_{\min }(E)=\frac{1}{2}(25+5 \sqrt{25+4 B(E)})
$$

Note that $P_{\min }$ reduces to $5 \sqrt{B}$ as $B$ grows large, as one would naively expect. The counts in the background $B$ for a given energy $E$ were determined by integrating the continuum of the spectra over $E \pm 1.5 \sigma(E)$, where the peak standard deviation $\sigma(E)$ was determined empirically by fitting the widths of the observed peaks to a quadratic function in energy.

The next step was to convert $P_{\min }(E)$ to a cross section by examining NRF peaks of known cross sections. For an NRF scattering measurement, the number of observed counts for a peak is

$$
N_{i}=n \cdot \phi(E) \cdot \varepsilon(E) \cdot \Delta \Omega \cdot \sigma_{i},
$$

where $n$ is the atoms $/ \mathrm{cm}^{2}$ of the target material, $\phi$ is the number of incident particles on the target, $\varepsilon$ is the detection efficiency, $\Delta \Omega$ is the solid angle of the detector and $\sigma$ is the cross section for the reaction. The linear relationship between the counts and cross section assumes that the counting rate is not saturated, which is valid for this size of target and integrated cross section. The subscript $i$ denotes quantities specific to the resonance, while the number of photons and detector efficiency are both functions of the photon energy. From a measurement on a known quantity of material with a known NRF cross section, one can determine the product of the number of photons, detector efficiency and detector solid angle. This product, which we define as $\alpha(E)$, is unique to the experimental setup, but can be applied to different isotopes within the same setup. For instance, if one can determine $\alpha$ from the ${ }^{12} \mathrm{C},{ }^{13} \mathrm{C}$ and ${ }^{27} \mathrm{Al}$ response using $\alpha=N_{i} / n \sigma_{i}$, then one can use $\alpha$ and $P_{\min }$ to determine the MDIC using $M D I C=P_{\min }(E) / \alpha(E) n$.

\footnotetext{
${ }^{1} 5 \sigma$ is the standard uncertainty threshold for declaring discovery of new phenomena in the nuclear and particle
} physics community 


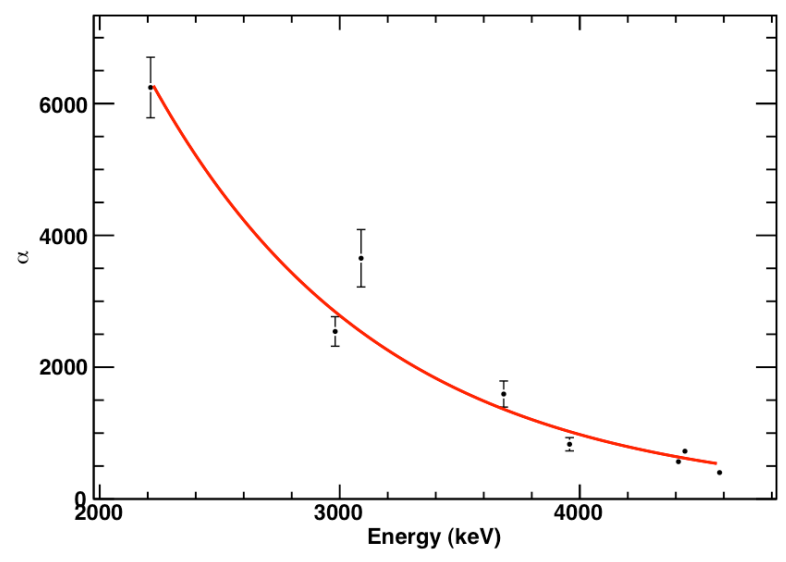

Figure 3.5. Determination of $\alpha(E)$. The points were determined from 8 NRF lines of ${ }^{12} \mathrm{C},{ }^{13} \mathrm{C}$ and ${ }^{27} \mathrm{Al}$. The red line is an exponential fit to those points.

simulations packages such as Geant4[Agnostinelli-03].

The 5.1 MeV end-point energy data on the HEU+Al was used to determine $\alpha(E) .{ }^{27} \mathrm{Al}$ has a number of lines that span a wide range in energy. In addition, two NRF lines from ${ }^{13} \mathrm{C}$ and one from ${ }^{12} \mathrm{C}$ provided additional points on which to determine $\alpha(E)$. The discrete values for $\alpha(E)$ for these observed NRF lines as well as an exponential fit to those points is shown in Figure 3.5.

Two MDICs were determined from the UCSB data, which are shown in Figure 3.6. The first was from the $4.3 \mathrm{MeV}$ end-point energy data. The second was from the sum of the 5.1 and $5.3 \mathrm{MeV}$ end-point energy data. Those two end-point energies are similar enough that the photon flux doesn't change significantly except for the highest energies, where a reliable MDIC cannot be determined because of a

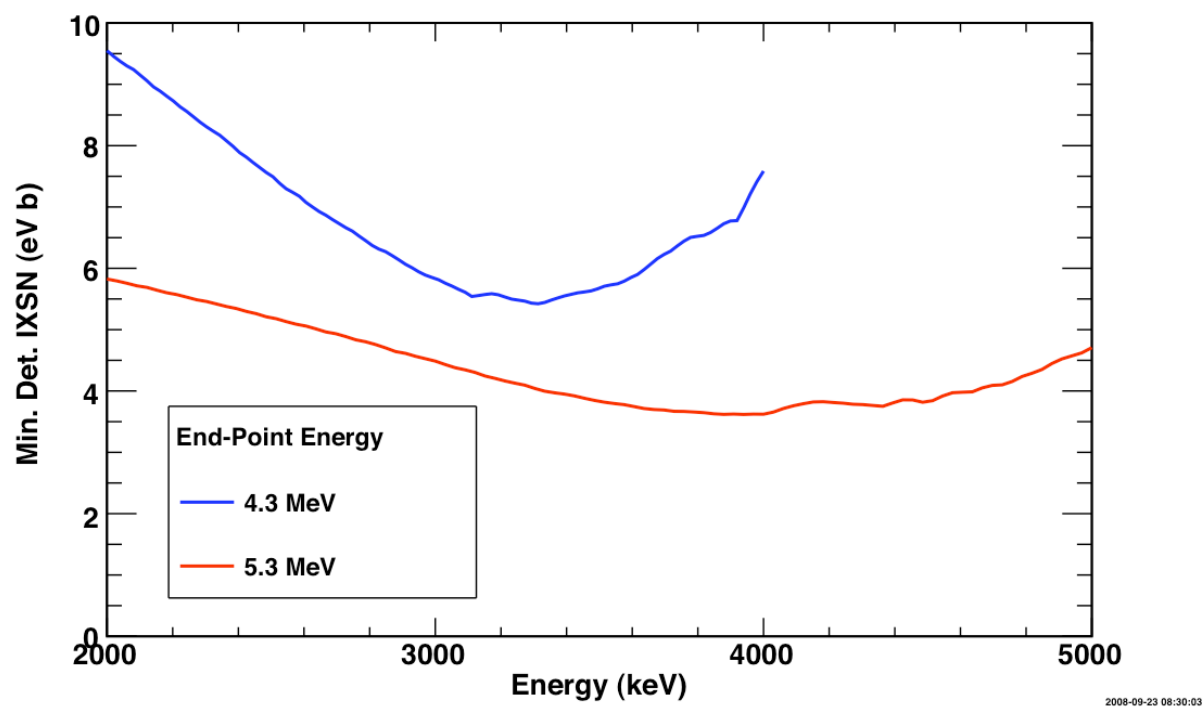

Figure 3.6. Minimum detectable integrated cross section from the UCSB measurements. 
lack of normalizing NRF lines above 4.5 MeV. For the 5.1 and 5.3 MeV MDIC, the $\alpha(E)$ function shown in Figure 3.5 was used after scaling for the total beam charge. For the $4.3 \mathrm{MeV}$ data, this $\alpha(E)$ was scaled by the ratio of the bremsstrahlung photon flux energy dependence for 4.3 and $5.3 \mathrm{MeV}$ end-point energies. The bremsstrahlung photon energy distributions were determined through Geant 4 simulations of the electron beam, radiator and collimator.

There are several observations on these plots of the MDIC:

- The minimum detectable integrated cross section is roughly $5 \mathrm{eV} \mathrm{b}$ from 2 to $5 \mathrm{MeV}$. Thus, if there are any undetected resonances, then they must have an integrated cross section of less than $\sim 5 \mathrm{eV} \mathrm{b}$.

- The MDIC for the $5.3 \mathrm{MeV}$ end-point energy data has a minimum around $4 \mathrm{MeV}$. This result is highly dependent on the sample material. For instance, lower $\mathrm{Z}$ materials would have lower optimum energy because of less background. In addition, the Compton edge from the resonance lines can product small bumps in an otherwise smooth distribution.

- While the 4.3 MeV MDIC has a lower optimum energy than the 5.3 MeV data, 3.3 MeV compared to $4 \mathrm{MeV}$, insufficient data at $4.3 \mathrm{MeV}$ was taken to improve on the MDIC from the $5.3 \mathrm{MeV}$ data.

It is worthwhile to determine the maximum count rate possible from a hypothetical resonance relative to the count rate from the known $1733-\mathrm{keV}$ resonance of ${ }^{235} \mathrm{U}$. This information is important for considering the possible benefits of a weaker high energy signal due to reduced attenuation through materials, such as shielding. We will assume the following:

- That the hypothetical resonance is at $4 \mathrm{MeV}$ with an integrated cross section of $4 \mathrm{eV} \mathrm{b}$. The energy of $4 \mathrm{MeV}$ was chosen because this energy corresponds to the minimum of the MDIC curve for the $5.3 \mathrm{MeV}$ end-point energy data. As the minimum, this energy at which the NRF measurement for this beam energy and target is most sensitive.

- The detector is a $90 \%$ relative efficiency HPGE and is $42 \mathrm{~cm}$ from the target.

- The target is $2 \mathrm{~mm}$ thick uranium enriched to $93 \%$.

- The number of incident photons per eV on resonance is the same for both the $1.733 \mathrm{MeV}$ and 4.000 MeV resonance. This assumption would likely translate into longer beam time measurements for the hypothetical $4 \mathrm{MeV}$ resonance because the higher photon beam energies produce higher background rates which in turn require a reduced beam current.

- There is 1 " lead filter for both measurements.

Given these assumptions, the ratio of the detector efficiency is 0.60 for the 4.0 and $1.7 \mathrm{MeV}$ resonances. This ratio includes the difference in attenuation due to the lead filter. The ratio of the resonant photons excited in the target and escaping out to the detector ${ }^{2}$ is 0.15 . This ratio is a little higher than the ratio of the cross sections, $4 / 30=0.13$ because of the reduced attenuation in the target material at $4 \mathrm{MeV}$. Based on these two ratios, any unobserved resonance will be at least 11 times weaker in count rate than the known 1733-keV resonance, assuming the same number of incident photons and a simple target geometry.

\footnotetext{
${ }^{2}$ This quantity is determined from the calculation of $C_{\text {thick }}$, discussed in Ref. [Metzger-59].
} 
Table 3.2. Amount of shielding material necssary to compensate for the minimum factor of 11 difference in count rate between the known $1733 \mathrm{keV}$ resonance and a hypothetical resonance at $4 \mathrm{MeV}$. The "Length" assumes the density shown in the second column.

$\begin{array}{crcr}\text { Material } & \begin{array}{r}\text { Density } \\ \left(\mathrm{g} / \mathrm{cm}^{3}\right)\end{array} & \begin{array}{c}\text { Length } \\ (\mathrm{cm})\end{array} & \begin{array}{c}\text { Thickness } \\ \left(\mathrm{g} / \mathrm{cm}^{2}\right)\end{array} \\ \mathrm{C} & 2.21 & 62.0 & 137.0 \\ \mathrm{Al} & 2.70 & 57.7 & 155.7 \\ \mathrm{Fe} & 7.87 & 24.6 & 193.4 \\ \mathrm{~Pb} & 11.35 & 32.0 & 363.3 \\ \mathrm{U} & 18.95 & 16.0 & 303.5\end{array}$

One argument for searching for resonances at higher energies is that the potential of greater penetration through shielding materials. This greater penetration occurs because of the reduced photon cross sections as the photon energy at higher energies in all but the highest $Z$ materials. Even if hypothetical higher energy resonances provided lower count rates for unshielded HEU, it is possible that the reduced attenuation for the higher energy signature through shielding could prove beneficial. Table 3.2 provides a list of material lengths required to compensate for the factor of 11 discussed above. It is important to keep in mind that the factor of 11 is based a hypothetical resonance with a strength is just below the measurement threshold to declare detection. Should new resonances be discovered, they will most likely be considerably smaller than this limit, thus requiring even more material to compensate for the difference in resonance strength relative to the known $1733 \mathrm{keV}$.

Implicit in this comparison is the use of a bremsstrahlung photon source. It is quite likely that a narrow bandwidth photon source, which would have considerably less background, could provide a more optimistic scenario for the exploitation of weak higher energy resonances.

\subsection{Search for Signatures between 5 and $9 \mathrm{MeV}$}

Several different measurements were conducted at TUD to search for NRF signatures above $5 \mathrm{MeV}$. The NRF signatures search for ${ }^{235} \mathrm{U}$ and ${ }^{238} \mathrm{U}$ were conducted using samples of HEU and DU. These measurements also included a boron sample for beam normalization. For both of these targets, bremsstrahlung photon beams with end-point energies of 8.3 and $10.0 \mathrm{MeV}$ were employed. In addition, measurements of a boron sample were conducted with an end-point energy of $10 \mathrm{MeV}$, and measurements on an empty container were conducted at both 8.3 and $10.0 \mathrm{MeV}$. Spectra from the $10 \mathrm{MeV}$ measurements on HEU, DU, boron and the blank container are shown in Figure 3.7. There are clearly many peaks in these spectra.

The observed peaks in the HEU spectrum originate through several different processes. Some of the peaks are from known NRF lines, such as the ${ }^{16} \mathrm{O}$ and ${ }^{11} \mathrm{~B}$ lines at 6917 and $7283 \mathrm{keV}$, respectively. Some of the peaks are from known radiative neutron capture, such as ${ }^{70} \mathrm{Ge}(\mathrm{n}, \gamma)$ line at $7414 \mathrm{keV}$. Some of the peaks appear in the HEU spectra as well as at least one other spectrum. These lines are labeled as "Not ${ }^{23}$ U." If these peaks appear in only the HEU and DU, it is likely that they are unknown $(n, \gamma)$ lines. The final group, labeled as "Unknown," appear only in the HEU spectrum spectra; these peaks are potentially new NRF lines of ${ }^{235} \mathrm{U}$.

The two unknown lines appear at 8210 and $9332 \mathrm{keV}$ in the $10-\mathrm{MeV}$ HEU spectrum. An expanded view of these peaks is shown in Figure 3.8. The $8210-\mathrm{keV}$ peak appears as a doublet with a likely $(\mathrm{n}, \gamma)$ 


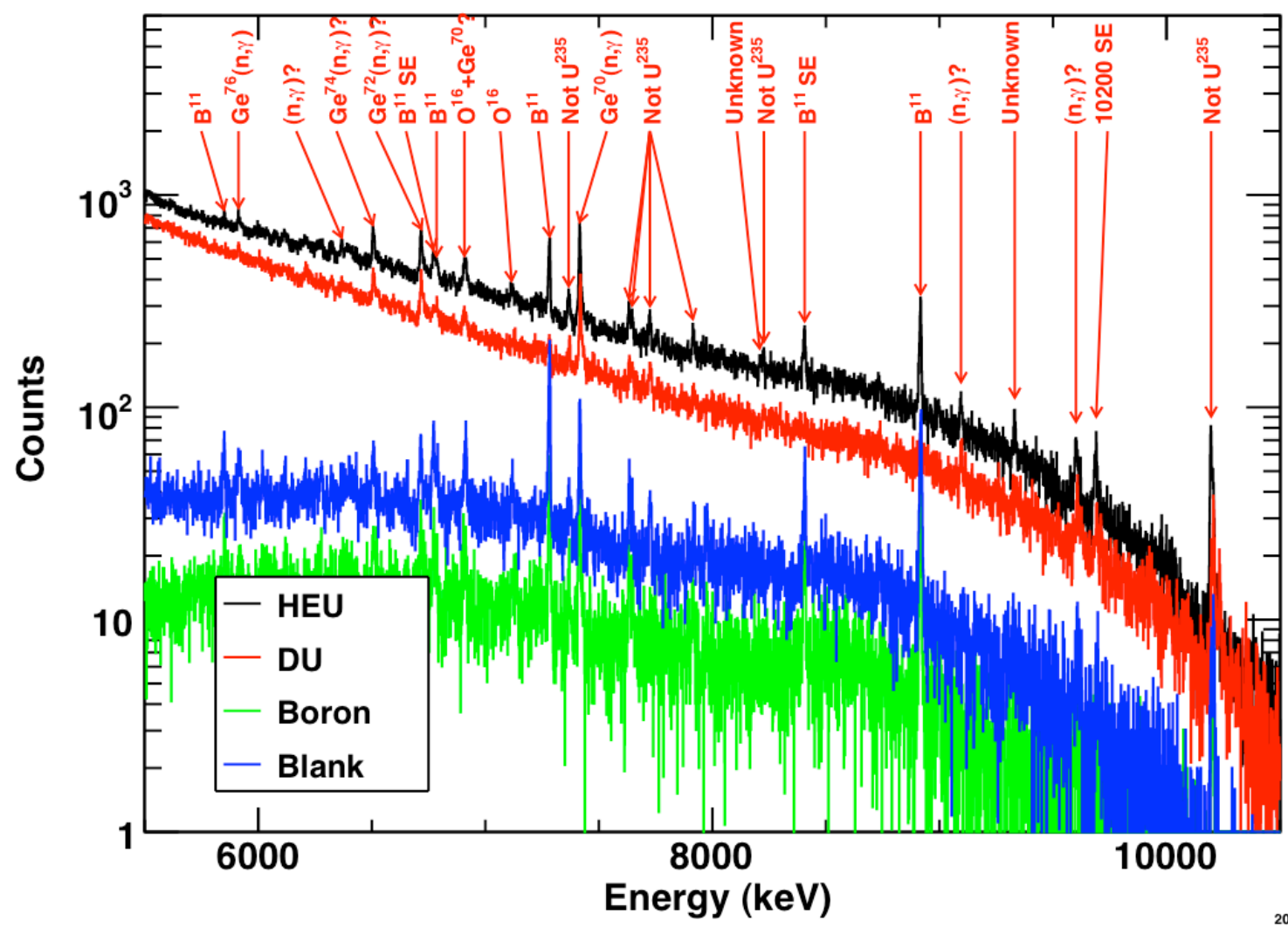

2008-11-03 14:06:

Figure 3.7. Spectra from $10 \mathrm{MeV}$ end-point energy measurements.

line; the $8225-\mathrm{keV}$ line is clearly present in the HEU as well as the DU spectrum at $10 \mathrm{MeV}$. There is no corresponding peak centered at $8210 \mathrm{keV}$ in the DU spectrum. A simultaneously fit to the 8210 and $8225-\mathrm{keV}$ peaks, using the same width for each peak, determined that the area of the $8210-\mathrm{keV}$ peak is $130 \pm 43$ counts.

The peak at $9331 \mathrm{keV}$ is clearly present in the $10 \mathrm{-MeV}$ spectra, with what appears to be hints of peaks in the DU data at $10 \mathrm{MeV}$ and the HEU and DU spectra at $8.3 \mathrm{MeV}$. A fit to this peak in the HEU at 10-

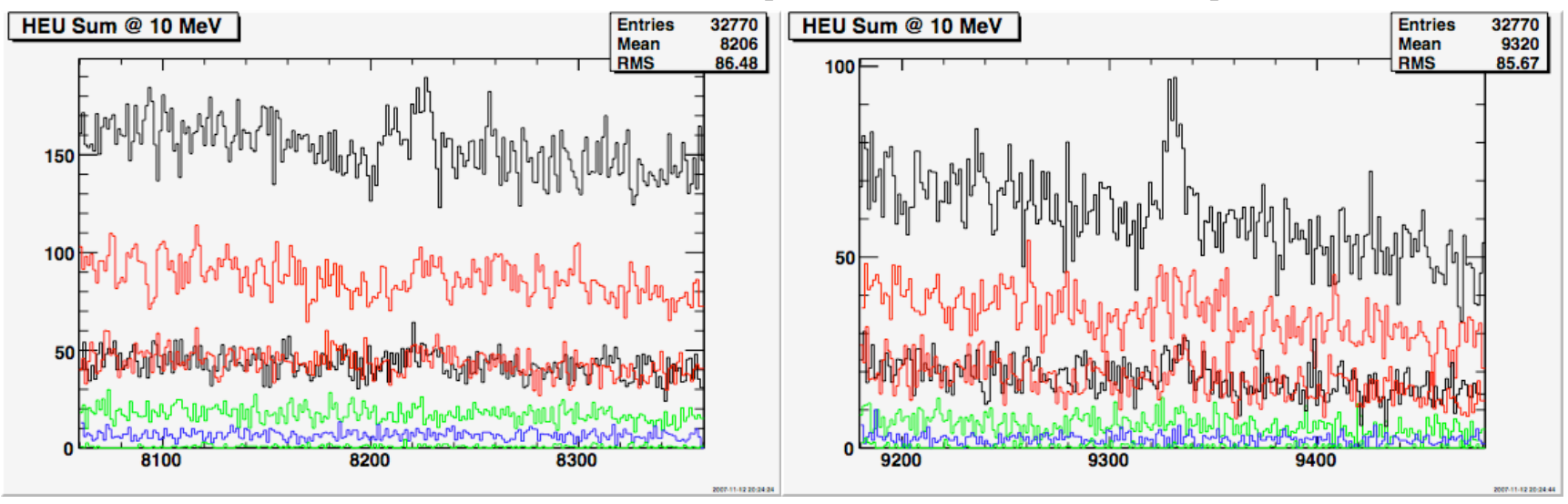

Figure 3.8. Expanded view of two peaks of unknown origin, left is at $8210 \mathrm{keV}$ and right is at $9331 \mathrm{keV}$. The lines spectra are HEU (black), DU (red), Blank (green) and Boron (blue). For target types with multiple lines, the top plot is at $10 \mathrm{MeV}$ and the bottom is at $8.3 \mathrm{MeV}$ for all types. 


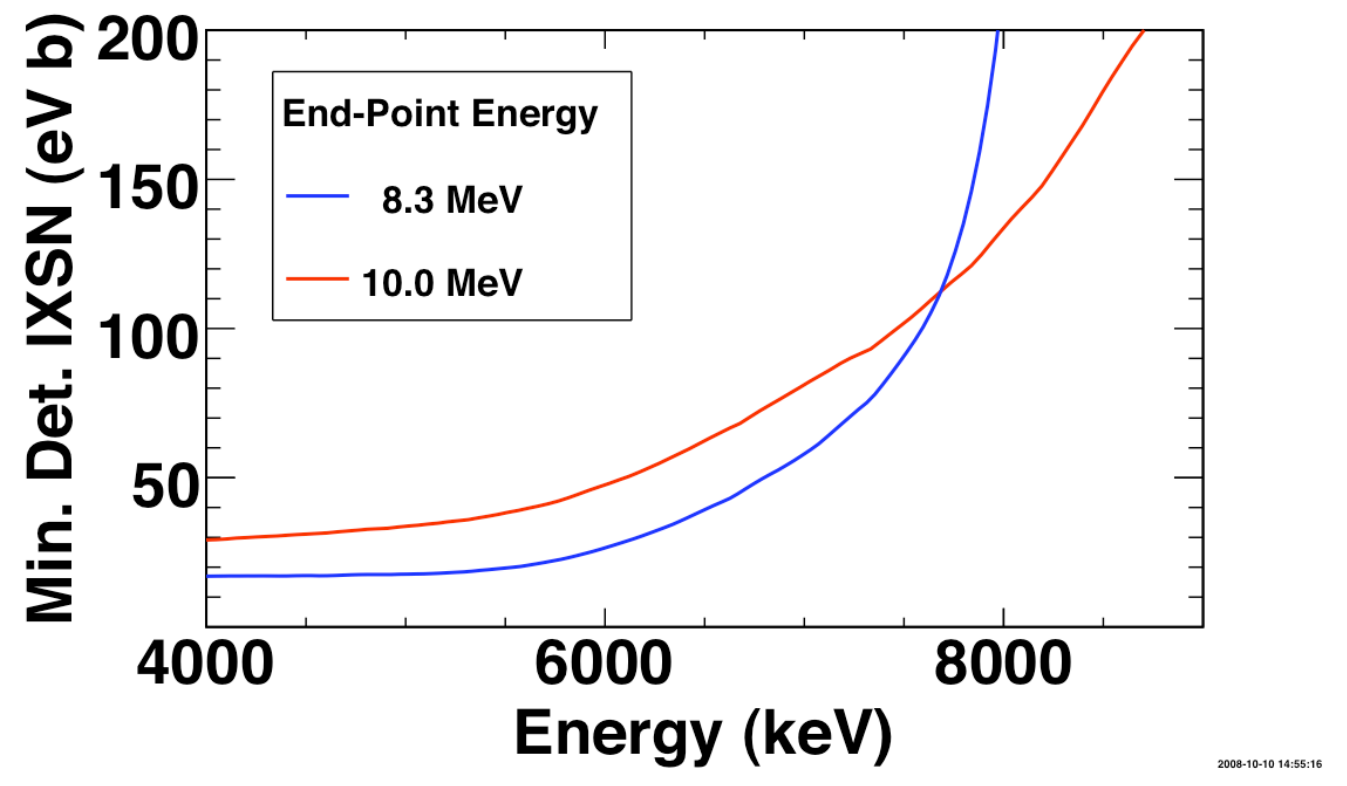

Figure 3.9. MDIC for TUD measurements.

$\mathrm{MeV}$ spectrum determines the peak area to be $234 \pm 40$ counts. A fit to the DU spectrum at $10 \mathrm{MeV}$, fixing the peak location and width determined from the fit to the HEU at $10-\mathrm{MeV}$ spectrum, determines the peak in the DU spectrum to be $59 \pm 23$. If part of the $9331-\mathrm{keV}$ peak strength is created by neutron capture processes, then the relative strength of the $9331-\mathrm{keV}$ peak should scale with other neutron-related processes observed in the spectrum. Scaling by the ratio of the $6505-\mathrm{keV}(\mathrm{n}, \gamma)$ line in the DU to HEU spectra, $0.618 \pm 0.080$, one can determine the net peak area at $9331 \mathrm{keV}$ due to non-neutron capture processes to be $139 \pm 54$ peak.

Any peak above the bremsstrahlung end-point energy, barring detector pileup and readout issues, is due to neutron radiative capture processes. There are peaks in the HEU and DU spectra for $8.3 \mathrm{MeV}$ near the 9331-keV peak observed in the $10-\mathrm{MeV}$ HEU data. Peak location and width determined from independent fits to these peaks in the $8.3-\mathrm{MeV}$ spectra agree within errors with the peak determined in the $10-\mathrm{MeV}$ HEU data. In addition, scaling the $8.3-\mathrm{MeV}$ peak size to account for difference in neutron exposure indicates that the $9331-\mathrm{keV}$ peak observed in the $10-\mathrm{MeV}$ HEU data could be entirely explained by neutron radiative capture.

Neither of these peaks is unambiguously attributed to an NRF response of ${ }^{235} \mathrm{U}$. Both peaks are too weak; neither approach the commonly accepted $5 \sigma$ threshold for declaration of new phenomenon. In addition, both peaks have background issues. The $8210-\mathrm{keV}$ peak is close to a possible $(\mathrm{n}, \gamma)$ peak, making the extraction of the peak challenging. The 9331-keV peak observed in the 10-MeV HEU data has no corresponding partner in the $10-\mathrm{MeV}$ DU data, but there appears to be peaks in both the HEU and DU data at $8.3 \mathrm{MeV}$.

The MDIC for ${ }^{235} \mathrm{U}$ was determined for the TUD data in a similar manner as for the UCSB data. The boron peaks served as the calibration between counts in the spectrum and the cross section to determine $\alpha(E)$. The MDIC for the two end-point energy measurements is shown in Figure 3.9. The sharp rise in 
the MDIC at high energies is likely due to the increased background at high energies from partial energy deposition of gamma rays generated through $(n, \gamma)$ processes, leading to events not restricted in energy by the bremsstrahlung end-point energy.

\subsection{Discussion on Additional ${ }^{235} \mathrm{U}$ Signature Search}

The lack of observed NRF signatures above $2 \mathrm{MeV}$ for ${ }^{235} \mathrm{U}$ and above $5 \mathrm{MeV}$ for ${ }^{238} \mathrm{U}$ raises two critical questions: is it worthwhile extending these measurements to reduce the MDIC and, if so, what can be done to improve the measurement sensitivity? While there are a number of possible ways to improve these measurements, we argue that from the point of view of applications such as detecting uranium isotopes using a bremsstrahlung photon source, the known signatures below $2 \mathrm{MeV}$ will likely provide a stronger signature than any currently undetected high energy resonance.

The UCSB and TUD uranium NRF signature search measurements were detector-rate limited. This limitation has a couple of important implications. First, the traditional dials one has to improve sensitivity in a scattering measurement, namely beam current, target size and detector solid angle (assuming that you are not increasing the number of detectors), cannot be modified to improve the measurement sensitivity. For instance, one might argue that one could improve sensitivity by increasing the size of the HEU sample. However, in this case one would have to either decrease the beam current and/or solid angle to maintain an acceptable counting rate. As a result, the measurement sensitivity would not be increased. In order to improve sensitivity, one must alter the rate of the desired signal to the total background rate.

The second implication of the detector-rate limitation is that higher energy signatures require larger cross sections to achieve the same sensitivity as lower energy signatures. For a fixed beam current, the background rate will increase as the end-point energy of the beam increases. The photon flux at the resonance will also increase, but not as quickly as the background. Thus, for fixed detector rate, NRF cross section and signal-to-noise, as the beam energy is increased the fraction of the signal rate to total background rate decreases. To maintain similar sensitivity, the resonance cross section must increase as the bremsstrahlung end-point energy increases.

The MDIC for the TUD measurement is an example of this decreased sensitivity as the end-point energy is increased. The $1733-\mathrm{keV}$ line of ${ }^{235} \mathrm{U}$ has an integrated cross section of $30 \mathrm{eV} \mathrm{b}$, and was easily observed in the $8 \mathrm{~h}$ measurement with a $2.2 \mathrm{MeV}$ end-point energy beam. In contrast, the MDIC in the TUD measurement was always greater than $18 \mathrm{eV}$ b after more than five times longer measurement times.

As a consequence of the detector rate limitation, it is likely that improved measurements would not identify new NRF signatures that would benefit applications of NRF where the measurement must be performed in a timely manner. After more than 40 hours, no signatures were observed in these measurements. For many potential applications, typical time scales are on the order of minutes, which would require three orders of magnitude improvement. Given the detector rate limitation, it is not likely that this level of improvement could be achieved using similar technology as in this experiment.

There are several improvements that could yield smaller MDIC to improve the measurement sensitivity. First, one can increase the number of detectors. One can also develop detectors that are capable of handling significantly higher counting rates. The filters could be optimized to improve low-energy rejection relative to the higher energy signal. Neutron shielding of the HPGe detectors would reduce the contribution of $(n, \gamma)$ lines which would improve sensitivity. Finally, the bremsstrahlung photon source could be modified to increase the number of high-energy photons relative total photon beam distribution. 
More exotic improvements could also improve sensitivity. Detectors with higher resolution than the existing HPGe detectors would significantly improve the signal-to-noise of any potential peak. Also, rather than conducting measurements with a bremsstrahlung photon source, a narrow bandwidth photon source could be employed, which would significantly reduce the background relative to the signal in the detector. Such a source is probably the single best way to improve sensitivities. 


\subsection{Source Comparison}

As NRF-based tools are designed for specific applications, the issue of the choice of photon source arises. We considered three general classes of photon sources with the appropriate energy. There are bremsstrahlung photon sources using high-duty factor electron accelerators (sometimes described as continuous wave or $\mathrm{CW}$ ), bremsstrahlung photon sources using pulsed linear accelerators, and narrow bandwidth photon sources using Compton backscattered photons. Other more exotic photon sources are under development, but we restrict our investigation to existing, energy-tunable sources. Our collaboration has conducted similar NRF measurements on each of these three types of sources to compare the performance of NRF-techniques using different photon sources.

To compare the performance of the different sources, similar NRF measurements on the 4842-keV line of ${ }^{208} \mathrm{~Pb}$ were conducted. All measurements were conducted on a $3.2 \mathrm{~mm}\left(1 / 8^{\prime \prime}\right)$ plate of natural lead. Lead was chosen as the target material for several reasons. There is significant experience handling lead, reducing the administrative effort to prepare for the measurements (in contrast to uranium). The similar atomic charge as uranium provides a comparable beam-related background rate. The large integrated cross section of the $4842-\mathrm{keV}$ line of ${ }^{208} \mathrm{~Pb}(3400 \mathrm{eV}$ b) enables fast, statistically significant measurements. Finally, the $\mathrm{CW}$ bremsstrahlung data on lead existed from prior efforts, so that there was no need to repeat that measurement.

The bremsstrahlung and narrow bandwidth sources use fundamentally different physics processes to generate the photons. The bremsstrahlung sources impinge an electron beam onto a metal foil. As the electrons strike that foil, photons with a continuos distribution of energies are created. The maximum energy of those photons is the electron beam energy, but the vast majority of photons are created at lower energies. A typical bremsstrahlung photon energy distribution for $5.3 \mathrm{MeV}$ electron beam is shown in Figure 4.1. In contrast, narrow bandwidth photon sources employ Compton backscattering to create a

\section{Photon Distributions}

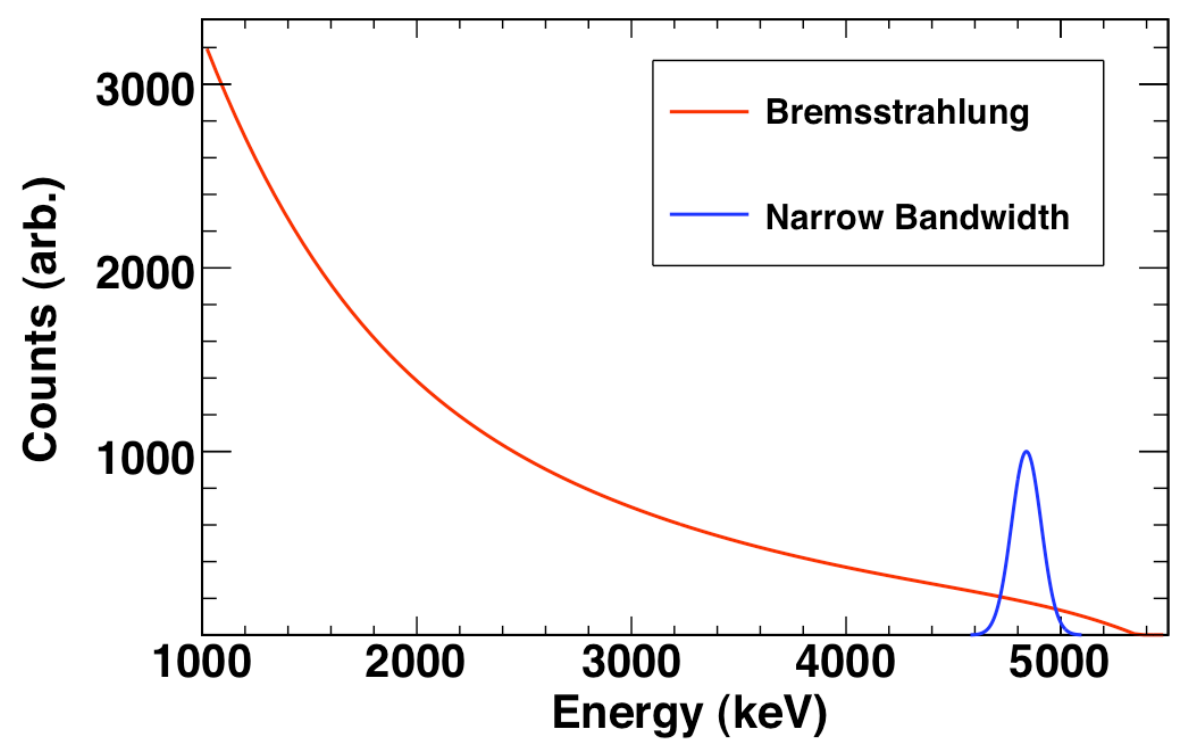

Figure 4.1. Comparison of energy distribution of $5.3 \mathrm{MeV}$ end-point energy bremsstrahlung distribution and 3\% FWHM narrow bandwidth distribution centered at $4.8 \mathrm{MeV}$. The relative scale of the two distributions is arbitrary. 
photon beam with a narrowly defined energy distribution. In this process, low energy photons are bounced off of extremely energetic electrons. This collision boosts the energy of the photons into the $\mathrm{MeV}$ range. For the case of the High Intensity Gamma Source (HIGS) at Duke University, the laser is $780 \mathrm{~nm}$ and the beam energy was $454 \mathrm{MeV}$ to generate $4.8 \mathrm{MeV}$ photons.

Each of the sources studied has certain advantages and disadvantages, as discussed below:

- CW Bremsstrahlung:

- Setup: CW bremsstrahlung systems use a high duty factor ${ }^{3}$ electron accelerator to generate a bremsstrahlung photon beam that is nearly always on.

- Advantages: the high duty factor nature of the beam reduces the instantaneous rate on the detector, reducing pileup issues in the data. In addition, bremsstrahlung sources are typically very bright photon sources.

- Disadvantage: A vast majority of the photon flux cannot contribute to the NRF signal, leading to wasted dose. There are only a few $\mathrm{CW}$ bremsstrahlung machines in the world. While they can be purchased commercially, they are moderately more expensive than their pulsed counterparts

- Pulsed Bremsstrahlung:

- Setup: pulsed bremsstrahlung sources use radio frequency linear accelerators (RF linacs) to create a beam of pulsed electrons. The duty factor of these machines is typically no more than $0.1 \%$.

- Advantages: these machines are much more common than $\mathrm{CW}$ accelerators, and they are moderately less expensive.

- Disadvantage: To maintain reasonable instantaneous detector rates, the low duty factor reduces the signal rate.

- Narrow Bandwidth:

- Setup: Laser light is backscattered off high-energy electron beam to create well-defined energy distribution of photons in the $\sim \mathrm{MeV}$ range.

- Advantages: Significantly higher fraction of beam is useful for generating signal, which improve the signal per dose. In addition, the well defined photon energies reduces the background under the signal as well as the overall detector rate

- Disadvantage: Source is strictly a laboratory facility due to its size and complexity.

This comparison of the photon sources is limited to the technical aspects of conducting the NRF measurements. Issues related to costs and operations are beyond the scope of this effort.

\subsection{Measurements}

The experimental setups were similar for each measurement. The measurements involved a shielded and collimated photon source, which was directed onto a $0.32 \mathrm{~cm}\left(1 / 8^{\prime \prime}\right)$ lead plate. A high purity germanium (HPGe) detector was placed at backward angles from the beam and was shielded by $10-20 \mathrm{~cm}$ on the five sides not directed toward the target. A lead filter, between $0.8-2.5 \mathrm{~cm}$ thick, was placed between the target and detector to reduce low energy beam-related background from the target relative to the higher energy signature. The $60 \mathrm{eV}$ recoil of the lead nucleus, which reduces the fluorescent photon energy by $60 \mathrm{eV}$ from the resonance, prevents the possible reabsorption of the fluorescent photon in the

\footnotetext{
${ }^{3}$ Duty factor is the fraction of time that the beam is on.
} 
Table 4.1. Properties of photon source comparison measurements. The relative detector efficiency compares the HPGe detector to a 3"x3" NaI detector at $25 \mathrm{~cm}$ using the $1.33 \mathrm{MeV}$ line of ${ }^{60} \mathrm{Co}$.

\begin{tabular}{|l|l|l|l|}
\hline & $\begin{array}{l}\text { CW } \\
\text { Brems. }\end{array}$ & $\begin{array}{l}\text { Narrow } \\
\text { Bandwidth }\end{array}$ & $\begin{array}{l}\text { Pulsed } \\
\text { Brems. }\end{array}$ \\
\hline Laboratory & UCSB & Duke/HIGS & ISU/IAC \\
\hline Beam Energy & $5.3 \mathrm{MeV}$ & $4.8 \mathrm{MeV}$ & $5.3 \mathrm{MeV}$ \\
\hline Tgt. Thickness & $0.3 \mathrm{~cm}$ & $0.3 \mathrm{~cm}$ & $0.3 \mathrm{~cm}$ \\
\hline Det. Rel. Eff. & $95 \%$ & $120 \%$ & $62 \%$ \\
\hline Tgt-to-Det Dist. & $91 \mathrm{~cm}$ & $25 \mathrm{~cm}$ & $62 \mathrm{~cm}$ \\
\hline Src-toTgt Dist. & $142 \mathrm{~cm}$ & N/A & $320 \mathrm{~cm}$ \\
\hline Filter Thickness & $2.5 \mathrm{~cm}$ & $0.8 \mathrm{~cm}$ & $2.5 \mathrm{~cm}$ \\
\hline Det. Angle & $110^{\circ}$ & $120^{\circ}$ & $129^{\circ}$ \\
\hline Det. Dead time & $20 \%$ & $36 \%$ & $2 \%$ \\
\hline Beam Current & $13 \mu \mathrm{A}$ & $62 \mathrm{k} / \mathrm{s}$ & $\begin{array}{l}1 \mathrm{nC} / \mathrm{burst} \\
\text { at } 600 \mathrm{~Hz}\end{array}$ \\
\hline Meas. Real Time & $88 \mathrm{~min}$ & $28 \mathrm{~min}$ & $574 \mathrm{~min}$ \\
\hline
\end{tabular}

lead. For all of these measurements, the entire beam passed through the target. A list of important experimental parameters is shown in Table 4.1.

The CW bremsstrahlung source measurements were conducted at the Free Electron Laser Facility at the University of California Santa Barbara (UCSB) in June 2006. This facility has an NRF measurement capability designed and built by Passport Systems with a 100\% duty factor peletron accelerator capable of a maximum electron beam energy of $\sim 5.3 \mathrm{MeV}$. The HPGe detector was surrounding by at least $20 \mathrm{~cm}$ $\left(8^{\prime \prime}\right)$ of lead on all sides except the side facing the target. The side facing the target had a $2.5 \mathrm{~cm}\left(1^{\prime \prime}\right)$ lead "filter", used to reduce the low-energy beam-related background from the target. The bremsstrahlung radiator consisted of $254 \mu \mathrm{m}$ tantalum on $1 \mathrm{~cm}$ thick water-cooled copper plate. The radiator was surrounded by $20 \mathrm{~cm}\left(8^{\prime \prime}\right)$ of lead, except for an approximately $2.4^{\circ}$ collimator along the electron beam axis, which defined the bremsstrahlung photon beam.

The pulsed bremsstrahlung source measurements were conducted at the Idaho Accelerator Center (IAC) of Idaho State University in April 2008. The IAC accelerator was capable of delivering up to $8 \mathrm{nC} /$ pulse at up to 600 pulses per second. The electron beam energy was $5.3 \mathrm{MeV}$. The detector shielding and radiator setup are similar to the UCSB setup. The radiator consisted of $4.2 \mathrm{~g} / \mathrm{cm}^{2}$ of tungsten. The resulting bremsstrahlung photon beam was collimated to a $1^{\circ}$ beam by a lead collimator in a $1.8 \mathrm{~m}$ thick concrete wall. One noticeable difference is the considerably longer source-to-target distance for the IAC measurement.

The narrow bandwidth source measurements were conducted at the High Intensity Gamma Source (HIGS) at Duke University in September 2007. The photon energy spread of the beam was approximately $3 \% \mathrm{FWHM}$ for the measurement. The well-defined beam allowed for considerably tighter measurement geometries than either of the bremsstrahlung measurements. In addition, less shielding around the detector was required, with only $10 \mathrm{~cm}\left(4^{\prime \prime}\right)$ on the upstream side of the detector and only $0.8 \mathrm{~cm}\left(5 / 16^{\prime \prime}\right)$ lead filter.

The pulsed structure of the IAC measurements limited the data rate on the detectors. The pulses were narrow compared to the pulse width in the HPGe detectors, a couple hundred nanoseconds compared to ten microseconds. Thus, if two photons from the same burst were detected, they generally fell 
immediately on top of each other in time. This high degree of overlap would make pileup rejection using digital signal processing a challenge. Instead, the detector rate was limited so that on average only 0.1 photons were detected per pulse. At this rate, the probability of detector pileup was $\sim 5 \%$, following Poisson statistics. The measurement setup achieved this counting rate by adjusting either the beam current or filter thickness (the detector was essentially immobile within the large shielding cave).

This rate on the detector has a number of interesting implications. First, NRF measurements using pulsed bremsstrahlung photon sources do not require fast HPGe detectors. The detector was running at about $60 \mathrm{~Hz}$. It is possible that fast detectors coupled with sophisticated digital signal processing may enable somewhat higher rates by employing sophisticated pileup rejection. One should be careful not to decrease detector volume with the goal of increasing detector rate; reduced detector volume has a disproportionate affect on the detection efficiency at the high energies of the NRF signatures.

\subsection{Results}

A comparison of the spectra from the three measurements is shown in Figure 4.2. The spectra have been normalized to have the same counts in the 4842-keV peak. The top plot of this figure shows the entire energy spectrum, while the bottom plot focuses on the resonance region.

There are many interesting qualitative observations to be made about Figure 4.2. The two bremsstrahlung measurements have similar spectra. The slight difference in the two spectra may be due to the larger detector angle of the pulsed bremsstrahlung measurement. One other difference is the significant $1022-\mathrm{keV}$ peak in the pulsed bremsstrahlung measurement. This peak is generated by pileup of two $511-\mathrm{keV}$ photons, so that it serves as a visual indicator of pileup. A striking difference in the bottom plot of Figure 4.2 is the difference in statistics of the pulsed bremsstrahlung measurements compared to the other two measurements despite the considerably longer pulsed bremsstrahlung measurement time. The narrow bandwidth source has considerably less background, as expected, than the bremsstrahlung measurements. It also has a large 1022-keV peak. A description of the quantitative comparison of these results follows.

\subsubsection{Counting Rate}

The three measurement setups were individually optimized to yield the highest count rates for each photon source. Measured count rates for the $4842-\mathrm{keV}$ resonance of ${ }^{208} \mathrm{~Pb}$ are shown in Table 4.2. The narrow bandwidth photon source measurement had the highest count rate; the clean, well-defined beam allowed for a significantly shorter target-to-detector distance as well as less shielding. The CW bremsstrahlung source count rate was roughly an order of magnitude slower, while the pulsed bremsstrahlung source count rate was two orders of magnitude below the $\mathrm{CW}$ measurements. This comparison of actual counting rates indicates the relative benefits of conducting optimized measurements on the different photon sources.

A second way to compare the counting rates is to normalize the different measurement setups to a common geometry. This comparison indicates which photon source provides the highest photon flux and would be useful in deciding which type of photon source to select when designing NRF-based applications. The CW bremsstrahlung measurement geometry was chosen as the nominal geometry. The other two measurements were corrected for differences in detection efficiency, lead filter, solid angle of the detector, and fraction of the photon flux contributing to the signal. 


\section{Comparison of Lead Spectra}
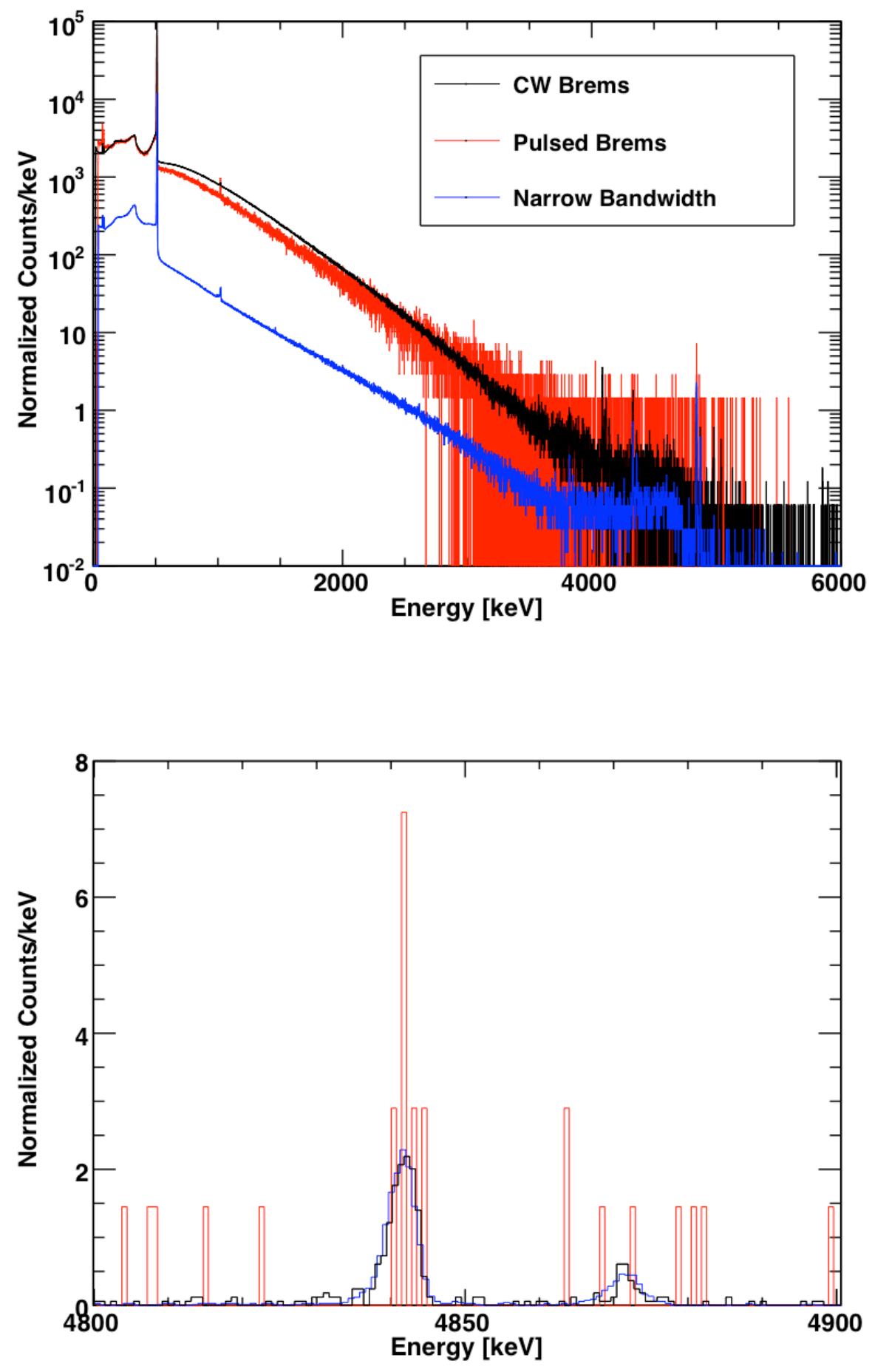

Figure 4.2. Comparison of NRF spectra on 1/8" lead using three different photon sources. The histograms have been normalized to have the same counts in the 4842-keV line of ${ }^{208} \mathrm{~Pb}$ as the pulsed bremsstrahlung source measurement. The top plot shows the full energy range, while the bottom plot is focused $\mathrm{n}$ the $4842-\mathrm{keV}$ resonance. 
Table 4.2. Summary of count rate results for different photon sources. See text for details.

\begin{tabular}{|l|l|l|l|l|l|}
\hline & $\begin{array}{l}\text { Count } \\
\text { Rate } \\
(\mathrm{cnts} / \mathrm{min})\end{array}$ & $\begin{array}{l}\text { Normalized } \\
\text { Count Rate } \\
(\mathrm{cnts} / \mathrm{min})\end{array}$ & $\begin{array}{l}\text { Signal/ } \\
\text { Noise }\end{array}$ & $\begin{array}{l}\text { Dose/ } \\
\text { Norm. } \\
\text { Counts } \\
(\mathrm{Rad} / \mathrm{cnts})\end{array}$ & $\begin{array}{l}\text { Dose to } \\
\text { Detection } \\
(\text { Rad })\end{array}$ \\
\hline CW Brems & 2.3 & 2.3 & $51.9 \pm 6.0$ & 13 & 65 \\
\hline Pulsed Brems. & 0.015 & 0.015 & $23.8 \pm 9.5$ & 11 & 66 \\
\hline $\begin{array}{l}\text { Narrow } \\
\text { Bandwidth }\end{array}$ & 28 & 0.52 & $145 \pm 12$ & 0.091 & 0.36 \\
\hline
\end{tabular}

Table 4.2 contains a summary of the corrections for the different geometries. The detector efficiencies at $4842 \mathrm{keV}$ were simulated in Geant4, using the detector dimensions provided in the detector specification sheets. The lead filter attenuation was determined from the total photon cross section through lead at $4842 \mathrm{keV}$, following the XCOM database [Berger-05]. The correction for the differences in solid angle is addressed in two pieces. First, the difference in solid angle for the different crystal sizes for a fixed distance from the target was incorporated into the detector efficiency simulations. Second, the

Table 4.3. Corrections for normalized

\begin{tabular}{|l|l|l|}
\hline \multicolumn{2}{c}{ counts } \\
\hline & $\begin{array}{l}\text { Pulsed } \\
\text { Brems. }\end{array}$ & $\begin{array}{l}\text { Narrow } \\
\text { Bandwidth }\end{array}$ \\
\hline Det. Eff. & 1.69 & 0.566 \\
\hline Filter & 1 & 0.43 \\
\hline$\Delta \Omega$ & 0.47 & 0.075 \\
\hline$\gamma$ Flux & 13.9 & 1 \\
\hline Total & 1 & 0.018 \\
\hline
\end{tabular}
difference in solid angle following the inverse of the square of the target-to-detector distance, shown as $\Delta \Omega$ in the table, is determined from the square of the target-to-detector distance. The final geometry correction, the photon flux density, was addressed by correcting for the ratio of the solid angle of the collimated beam on target (with the origin at the radiator). For the narrow bandwidth measurement, this correction was set to one because the photon flux impinging on the target was measured directly, unlike the bremsstrahlung measurements in which the electron beam flux, not the photon beam flux, was measured.

All measurements were limited by the detector rate. As a result, it is not possible to increase the signal of the detector. For instance, one could move the target closer to the radiator in the pulsed bremsstrahlung measurement to match the $\mathrm{CW}$ bremsstrahlung measurement, but then one would have to reduce the beam current or decrease the detector solid angle in order to bring detector rate back down to a nominal operating limit. The geometrical corrections can reduce the normalized counting rate, but they cannot increase it. As a result, normalization of the pulsed bremsstrahlung measurement is set to 1 despite the product of the correction factors being larger than 1 .

The resulting normalized count rates are listed in Table 4.2. After correcting for geometrical differences, the $\mathrm{CW}$ bremsstrahlung measurement has the highest rate, followed by the narrow bandwidth source and then the pulsed bremsstrahlung source. These results indicate the relative photon flux on resonance for each of these photon sources. These observations, however, are based on current technologies. It is unlikely that the normalized pulsed bremsstrahlung rate will ever significantly improve relative to the $\mathrm{CW}$ bremsstrahlung rate, however it is quite conceivable that improvements in the technology of the narrow bandwidth source could increase the normalized count rate to beyond that of the CW bremsstrahlung. 


\subsubsection{Signal-to-Noise}

Results from the analysis of the signal-to-noise $(\mathrm{S} / \mathrm{N})$ are shown in Table 4.2. The signal-to-noise was determined by conducting a region-of-interest analysis. This analysis involves counting the events in the peak region and two background regions on either side of the peak, and appropriately weighing and subtracting these counts to get signal and background counts. The narrow bandwidth measurements provide a 3 times better signal-to-noise than the $\mathrm{CW}$ bremsstrahlung, which in turn provides a 2 times better signal-to-noise than the pulsed bremsstrahlung.

These quantitative comparisons are specific to the experimental setups of these measurements. However, one can draw two general conclusions from these results. First, the narrow bandwidth source provides an improved $\mathrm{S} / \mathrm{N}$ compared to the bremsstrahlung sources. This result is not surprising considering the difference in the incident photon distribution. Second, some effect beyond the incident photon energy distribution is reducing the $\mathrm{S} / \mathrm{N}$ of the pulsed bremsstrahlung measurement relative to the $\mathrm{CW}$ bremsstrahlung measurement.

One possible explanation for the latter observation is the difference in detector pileup for the two bremsstrahlung source measurements. Pileup can impact the data in two ways. It can remove events from a peak in the spectra, and it can increase the observed energy of a nominal background event. Both of these processes will reduce the $\mathrm{S} / \mathrm{N}$.

We examined the potential impact of pileup on the $\mathrm{S} / \mathrm{N}$ in the pulsed bremsstrahlung measurement. Initially, we assumed that the observed spectrum from the $\mathrm{CW}$ bremsstrahlung measurements represents a measurement without pileup (this assumption is not strictly true, but we later correct for this assumption). For a given pileup probability $P_{p}$, we constructed a hypothetical spectrum of $N$ events from two components. The first component represents the non-pileup contribution and contains a total of $N\left(1-P_{p}\right)$ events. This component is simply a scaled version of the $\mathrm{CW}$ bremsstrahlung source spectrum. The second component represents the pileup contributions and contained a total of $N P_{p}$ events. The pileup contribution was generated by histogramming the sum of two energies randomly selected from the nonpileup energy distribution. These constructed histograms were used to study the impact of pileup on the signal-to-noise ratio.

The ratio of the $1022-\mathrm{keV}$ peak to $511-\mathrm{keV}$ peak is a sensitive indicator of the pileup. Using the constructed histogram discussed above, the ratio of these two peaks tracked with the pileup probability used to generate the constructed histograms, as seen in Table 4.4. We observed that $P_{p}=0.049$ provides the same ratio of 1022 to 511 in the constructed spectrum as in the pulsed bremsstrahlung spectrum. For this pileup probability, the $\mathrm{S} / \mathrm{N}$ for the $4842-\mathrm{keV}$ line of the simulated pileup spectrum agrees with that of the pulsed bremsstrahlung spectrum. This agreement strongly suggests that the difference in $\mathrm{S} / \mathrm{N}$ of the $\mathrm{CW}$ and pulsed bremsstrahlung is due to the difference in pileup in the detector.
Table 4.4. Pulse Pileup Study. The final row is for the values for the measured spectrum from the IAC. See text for more details.

\begin{tabular}{|c|c|c|}
\hline $\mathrm{P}_{\mathrm{p}}$ & $1022 / 511$ & $4842 \mathrm{~S} / \mathrm{N}$ \\
\hline 0.00 & $0.00113 \pm 0.00001$ & $52 \pm 6$ \\
\hline 0.01 & $0.00226 \pm 0.00002$ & $43 \pm 5$ \\
\hline 0.03 & $0.00469 \pm 0.00003$ & $31 \pm 3$ \\
\hline 0.049 & $0.00714 \pm 0.00004$ & $27 \pm 3$ \\
\hline 0.10 & $0.0139 \pm 0.0001$ & $15 \pm 1$ \\
\hline 0.20 & $0.0300 \pm 0.0001$ & $8 \pm 1$ \\
\hline IAC & $0.0072 \pm 0.0002$ & $24 \pm 10$ \\
\hline
\end{tabular}

The CW bremsstrahlung spectrum included a moderate amount of pileup. Using the ratio of the 1022$\mathrm{keV}$ and $511-\mathrm{keV}$ peaks, it was determined that the pileup probability in this spectrum was about $1 \%$. 
Thus, the actual pileup probability for the pulsed bremsstrahlung spectrum is $5.9 \%$ after correcting for this initial contribution to the pileup.

A simple check of the rates following Poisson statistics supports this determination of the pileup probability. The observed counting rate was 0.109 counts per beam burst. Following Poisson statistics, this implies a mean event rate of 0.115 events per burst. The mean event rate is larger than the observed rate because multiple events are contained within pileup events. Following Poisson statistics for this mean event rate, there is a $5.4 \%$ chance of pileup events. This pileup probability agrees well with the pileup probability determined through the constructed pileup histogram approach discussed earlier. This agreement supports the validity of the observation of the strong impact of the pileup events on the signalto-noise of the NRF seen through the histogram approach.

One can conclude that the higher fraction of pileup events in the pulsed bremsstrahlung measurement causes the lower signal-to-noise ratio compared to the $\mathrm{CW}$ bremsstrahlung measurement. This observation is an important distinction between pulsed and CW bremsstrahlung NRF measurements; it is created by driving the detector (instantaneously) at significantly higher rate for the pulsed bremsstrahlung measurements than for the $\mathrm{CW}$ bremsstrahlung measurement.

The signal-to-noise ratio of the $4842-\mathrm{keV}$ peak of ${ }^{208} \mathrm{~Pb}$ for the pulsed bremsstrahlung source is illustrative of the challenges to be faced when conducting NRF measurements with a pulsed bremsstrahlung system. There are no physics reasons why the energy distribution of photons exiting the lead target is any different for the CW bremsstrahlung source than for the pulsed bremsstrahlung source. The observed differences in the spectrum must therefore come from how those two energy distributions are observed, which ultimately is controlled by the experimenters.

\subsubsection{Dose Per Counts}

One of the attractive features of the narrow bandwidth source is a reduction in the dose delivered to the sample compared to bremsstrahlung photon source. To examine this potential, the absorbed dose in water was determined for both the narrow bandwidth source and the bremsstrahlung source (the $\mathrm{CW}$ bremsstrahlung source and pulsed bremsstrahlung source will deliver the same dose for the same total electron beam charge). From this dose and the observed spectra, the dose per normalized counts (as discussed above) was determined. The results are listed in Table 4.2.

The qualitative dependence of the dose per counts for the different photon sources is not surprising. The narrow bandwidth source provides considerably lower dose than the bremsstrahlung sources, while the two bremsstrahlung sources provide similar counts/dose.

The dose per counts for the narrow bandwidth source appears on the surface not to deliver as low of a dose as one might have hoped was possible for this type of source. This higher-than-expected dose is due to the relatively wide energy distribution of the narrow bandwidth source, a FWHM of $160 \mathrm{keV}$. This width is a roughly a factor of $2 \cdot 10^{4}$ larger than the resonance itself, so that one could theoretically reduce the dose per count from $90 \mathrm{mRad} /$ count down to $5 \mu \mathrm{Rad} /$ count. Such an extreme reduction in the width of the energy distribution is likely not practical, as the bandwidth will need to be broad enough to allow for variations over time in the energy of the photons to still strike the desired resonance region. It is more likely that future narrow bandwidth sources will be $1-10 \mathrm{keV}$ wide, which would reduce the dose/count down to 0.6 to $6 \mathrm{mRad} /$ count. While this is a considerable reduction in dose compared to the bremsstrahlung sources, it is still significantly higher than would be practical for a system that conducts repetitive screening. In addition, one should also note that these dose/counts are for the $4842-\mathrm{keV}$ line of 
${ }^{208} \mathrm{~Pb}$, which is an unusually strong NRF response. Doses for detection of other weaker NRF peaks may be significantly larger.

It is also beneficial to compare dose for detection. The number of counts required to declare detection will depend on the $\mathrm{S} / \mathrm{N}$ ratio. Following hypothesis testing, it can be shown that the number of counts required to declare detection is

$$
N=\left(\frac{\alpha+1}{\alpha}\right)^{2}\left(A_{P D}+\sqrt{\frac{1}{1+\alpha}} A_{F A}\right)^{2},
$$

where $\alpha$ is the $\mathrm{S} / \mathrm{N}$ ratio, $A_{P D}$ is the number of standard deviations below the mean of the threat population the threshold must be set to achieve the specified probability of detection, and $A_{F A}$ is the number of standard deviations above the mean the threshold must be located to achieve the specified false alarm probability. For illustrative purposes, we set the probability of detection to $95 \%$ and the false alarm probability to $0.1 \%$, which correspond to $A_{P D}=1.6$ and $A_{F A}=3.1$. The counts to declare detection as a function of $\mathrm{S} / \mathrm{N}$ are shown in Figure 4.3. For the $4842-\mathrm{keV}$ peak of ${ }^{208} \mathrm{~Pb}$, one then requires 6,5 and 4 counts to declare detection using the pulsed bremsstrahlung source, $\mathrm{CW}$ bremsstrahlung source and narrow bandwidth source, respectively. For lower $\mathrm{S} / \mathrm{N}$, the relative difference between the counts required may be significantly larger.

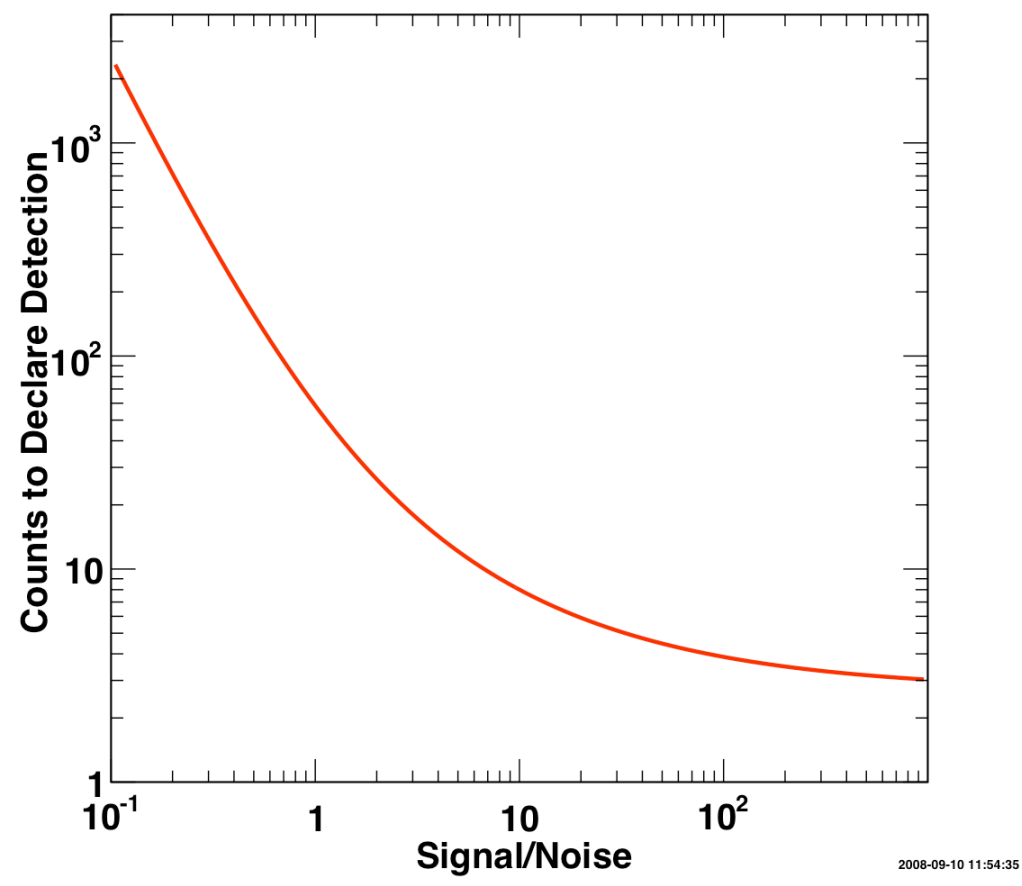

Figure 4.3. Counts to detection as a function of the $\mathrm{S} / \mathrm{N}$ ratio for $A_{P D}=1.6$ and $A_{F A}=3.1$. 


\subsection{Summary}

NRF measurements were conducted on lead targets using three different photon sources. Those sources were a CW bremsstrahlung source, a pulsed bremsstrahlung source and a narrow bandwidth source. From our experimental observations, we conclude that:

- In terms of raw counting rates possible, the narrow bandwidth source had the highest count rate; the clean beam of the HIGS facility allowed for considerably tighter geometries than for the bremsstrahlung measurements.

- Normalizing the count rates from the different sources to reflect measurements for the same physical measurement geometry, the $\mathrm{CW}$ bremsstrahlung source provided the highest count rate. This conclusion is based on current technology. It is possible that future narrow bandwidth sources, which may be brighter, will provide higher photon fluxes.

- The narrow bandwidth source provides for significantly higher $\mathrm{S} / \mathrm{N}$ ratios than the bremsstrahlung sources. However, the $\mathrm{S} / \mathrm{N}$ from all three sources for this resonance are very high $(>20)$, so that there is little practical benefit to the improved $\mathrm{S} / \mathrm{N}$ ratio of the narrow bandwidth source for this resonance. This observation is based on the $4842-\mathrm{keV}$ line of ${ }^{208} \mathrm{~Pb}$ which is intrinsically extremely strong. For other lines, such as the $1733-\mathrm{keV}$ of ${ }^{235} \mathrm{U}$, the $\mathrm{S} / \mathrm{N}$ ratio is $\sim 1$ for a bremsstrahlung source, so that an improvement in $\mathrm{S} / \mathrm{N}$ using the narrow bandwidth source may significantly reduce statistical requirements.

- Measurements on the pulsed bremsstrahlung system are limited by pileup concerns to about 0.1 counts per beam pulse. This limitation greatly restricts the potential counting rate. For instance, for a $1 \mathrm{kHz}$ repetition rate $\mathrm{RF}$ linac, one is limited to $\sim 100 \mathrm{~Hz}$ detection. In contrast, with a $\mathrm{CW}$ bremsstrahlung source, detector rates in the 10's of $\mathrm{kHz}$ are routinely achieved. For this reason, in the foreseeable future NRF measurements with pulsed bremsstrahlung sources will be limited in counting rate to about $1 / 100$ of that using a CW bremsstrahlung source. Thus, measurements that take minutes with a $\mathrm{CW}$ bremsstrahlung source will take hours with a pulsed bremsstrahlung source. The only way to improve this performance is to dramatically increase the repetition rate of the accelerator for the pulsed bremsstrahlung source. Note that the time structure must be larger than the integration times of the signal from the detector, $\sim 10 \mu \mathrm{s}$, to make a difference without digital signal processing.

- At $60 \mathrm{~Hz}$ counting rate for the pulsed bremsstrahlung source, one does not require a fast detector.

- The pileup in the detector for pulsed bremsstrahlung sources can dramatically affect the $\mathrm{S} / \mathrm{N}$ ratio. For instance, the $5 \%$ pileup rate observed in the IAC measurements decreased the $\mathrm{S} / \mathrm{N}$ of the $4842-\mathrm{keV}$ peak of ${ }^{208} \mathrm{~Pb}$ by a factor of 2 !

- The absorbed dose in water when trying to detect the 4842-keV line of $\mathrm{Pb}$ is significantly lower for the narrow bandwidth source than for the bremsstrahlung sources. However, even after correcting for significantly reduced bandwidth for possible future systems, the dose is in the milli-Rad range, which significantly limits the possible applications of the technique. 


\subsection{Measurements at HIGS}

A series of NRF-related measurements were conducted at the HIGS during September 2007. The measurements consisted of NRF measurements of lead and depleted uranium, as well as a photon scattering Z-dependence study. The lead measurement has already been extensively discussed in comparison to measurements using other NRF sources. In this section, the NRF measurements of DU and the $\mathrm{Z}$ study will be discussed.

NRF measurements at HIGS were conducted on a $2 \mathrm{~mm}$ thick DU plate. The geometry of the DU measurement was the same as the lead measurements discussed earlier. These measurements using a narrow bandwidth source can be compared to similar measurements conducted by Passport Systems using a CW bremsstrahlung photon source. The DU for the CW bremsstrahlung source measurement was $1 \mathrm{~cm}$ thick. The spectra of the resonance region from these two measurements are shown in Figure 5.1. The live times for the spectra shown in this figure are 6.4 hours for the HIGS measurements and 0.4 hours for the CW bremsstrahlung measurement.

The passive background in the narrow bandwidth source data was subtracted, reducing the non-resonant background by roughly a factor of two. It is appropriate to compare the background-subtracted HIGS spectra with non-background-subtracted CW bremsstrahlung spectra because of how the background was sampled. The HIGS photon beam is pulsed with a period of roughly $180 \mathrm{~ns}$, which is short on the time scale relevant to the HPGe detector response. Unfortunately, the data acquisition system was not gated on the beam pulses, so that background was continuously. Subtracting the background from the HIGS spectra therefore corrects for this disproportionate sampling of the background.

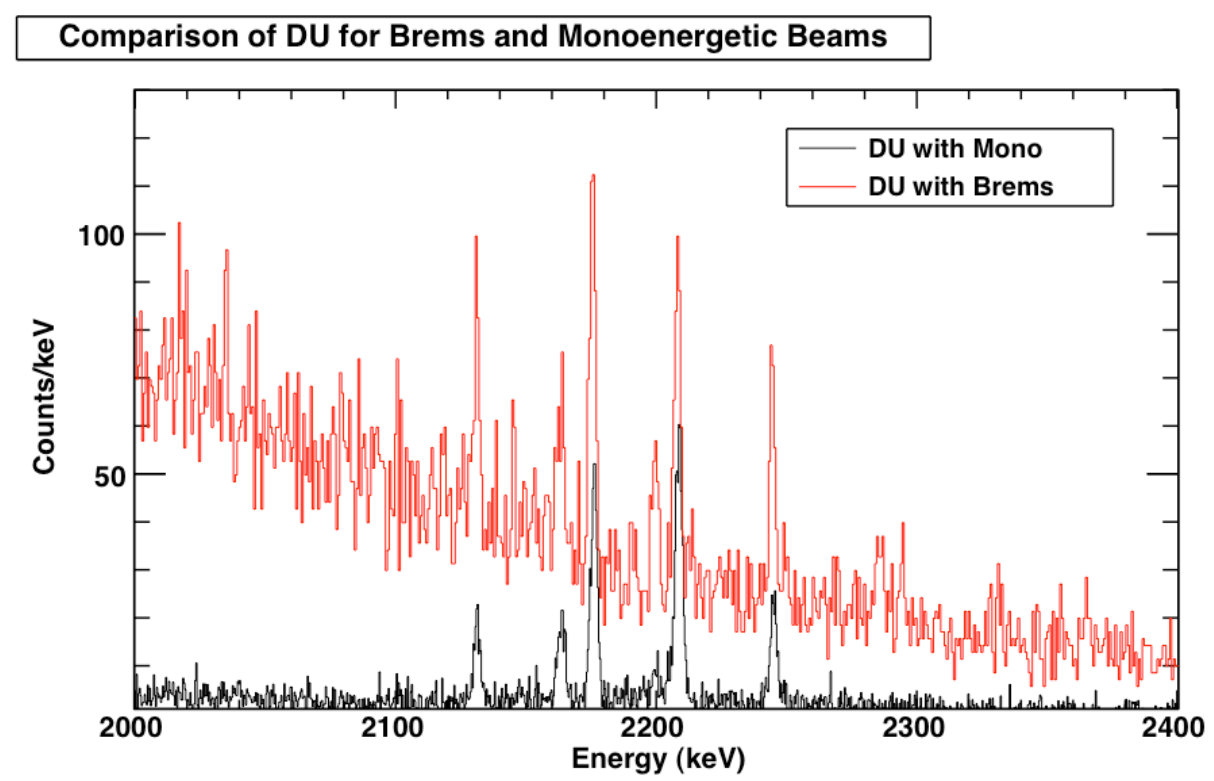

2008-09-08 16:08:20

Figure 5.1. NRF spectra of DU from a CW bremsstrahlung source (red) and a narrow bandwidth source (black). The passive background in the DU measurement has been removed, which reduces the non-resonant background by a factor of $\sim 2$. The CW bremsstrahlung data was taken by Passport System, Inc. 


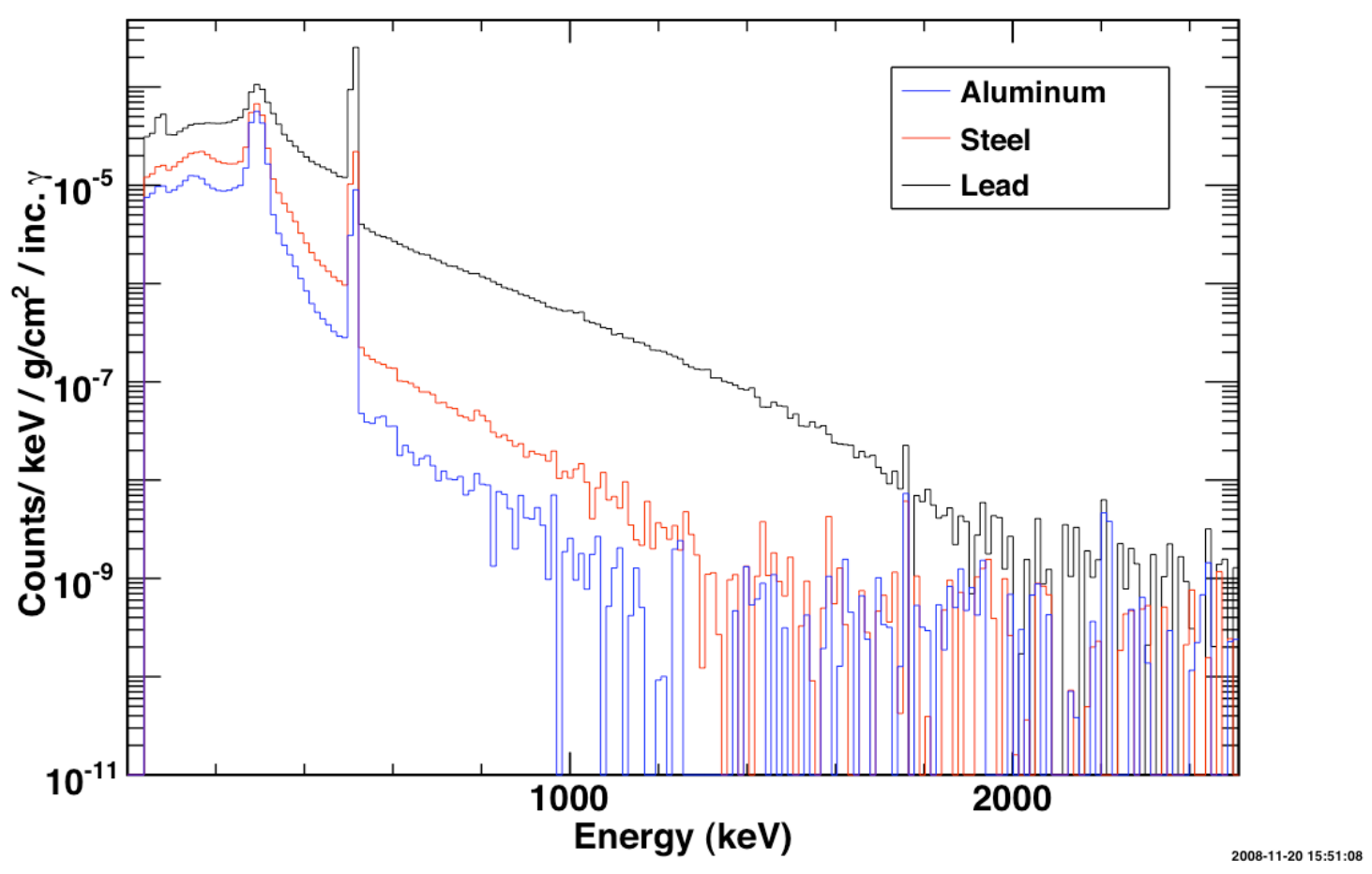

Figure 5.2. Spectra from Z-study of photon scattering. See text for details.

It is clear from Figure 5.1 that the narrow bandwidth source provides a significant improvement in the $\mathrm{S} / \mathrm{N}$ ratio. With the passive background subtracted, the $\mathrm{S} / \mathrm{N}$ of the HIGS measurement is roughly a factor of 7 times larger than for $\mathrm{CW}$ bremsstrahlung measurement. Without the passive background subtracted, this ratio drops by a factor of two. These improvements incorporate the correction for the difference in detector resolution for the two measurements.

The $\mathrm{S} / \mathrm{N}$ ratio of the DU NRF response agrees roughly with expectations based on coherent elastic scattering cross sections. According to Rullhusen et al. [Rullhusen-82], the coherent elastic cross section on uranium at $120^{\circ}$ for $2-\mathrm{MeV}$ photons is $80 \mu \mathrm{b} / \mathrm{Sr}$. This strength can be compared to the $57 \mathrm{eV} \mathrm{b}$ integrated cross section of the 2209-keV NRF line of ${ }^{238} \mathrm{U}$. Assuming that the NRF response is isotropic and integrating the elastic cross section over the observed peak width, one would expect a $\mathrm{S} / \mathrm{N}$ ratio of 15 . This result is in reasonable agreement with the observed $\mathrm{S} / \mathrm{N}$ ratio of 11 (which includes the passive background subtraction).

A comparison of the spectra from the Z-study in photon scattering is shown in Figure 5.2. Such a study is useful to begin to develop an understanding of possible background contributions in NRF measurements using narrow bandwidth sources. The materials consisted of $4 \mathrm{~mm}$ aluminum, $3 \mathrm{~mm}$ steel and $3 \mathrm{~mm}$ lead. For this study, photon spectra were measured in the same geometry as the NRF measurements at HIGS with a $2.2 \mathrm{MeV}$ beam, with the exception that there was no filter over the front face of the detector. The spectra have been normalized for photon flux and moles $/ \mathrm{cm}^{2}$. The spectra have been corrected for passive room background contributions, but not for beam-related target-independent contributions. 
Table 5.1. Comparison of Compton peak and $511-\mathrm{keV}$ peak strengths in the Z-study for photon scattering.

\begin{tabular}{|l|l|l|l|}
\hline Material & $\begin{array}{l}511-\mathrm{keV} \text { peak } \\
\text { Counts }\end{array}$ & $\begin{array}{l}\text { Compton peak } \\
\text { counts }\end{array}$ & 511/Compton \\
\hline Aluminum & $1.3 \cdot 10^{6}$ & $1.8 \cdot 10^{7}$ & 0.072 \\
\hline Steel & $3.3 \cdot 10^{6}$ & $2.2 \cdot 10^{7}$ & 0.15 \\
\hline Lead & $3.5 \cdot 10^{7}$ & $4.2 \cdot 10^{7}$ & 0.83 \\
\hline
\end{tabular}

There are four notable features of these spectra:

1. There is no obvious "bump" around the beam energy to suggest detection coherent elastic scattering.

2. The continuum above the $511-\mathrm{keV}$ peak shows strong dependence on the $\mathrm{Z}$ of the target material.

3. The 511-keV peak also varies with $\mathrm{Z}$ of the target material.

4. The peak around $300 \mathrm{keV}$ is from Compton scattering of the $2.2 \mathrm{MeV}$ photon beam on the target material and scattered into the detector at $120^{\circ}$. The amplitude of this peak is correlated with the target material $\mathrm{Z}$.

A quantitative comparison of the 511-keV peak and Compton peak is shown in Table 5.1. The counts in the $511-\mathrm{keV}$ peak were determined by conducting a region-of-interest analysis using $1.5 \sigma$ window. The Compton peak counts were also determined through a region-of-interest analysis, but using the fullwidth at half-maximum to define the window. While the 511-keV peak and the Compton both individual demonstrate a correlation of the target material $\mathrm{Z}$, the ratio of the two provide a more robust result largely reducing effects such as target thickness. A more thorough study would need to be completed to examine issues such as dependence on target thickness and layering of different materials to develop a solid understanding of the potential of this method to characterize the $Z$ of a sample.

In summary, the measurements at HIGS provided much new information. The measurements demonstrated the significant advantage of a narrow bandwidth source for improved signal-to-noise of the NRF response of DU. One would expect similar advantages for HEU. In addition, a study of the 511$\mathrm{keV}$ peak and Compton peak scattered from a set of materials demonstrated a potential sensitivity to $Z$. 


\subsection{References}

[Agnostinelli-03] S. Agostinelli et al., "Geant4 - a simulation toolkit," Nucl. Instr. Meth. A, vol. 506, p. 250 (2003).

[Alston-68] W.J. Alston III, H.H. Wilson and E.C. Booth, "Resonance Fluorescence and Absorption Measurements of Transition Strengths in Odd-Mass Nuclei," Nucl Phys. A116, 281-288 (1968). See also Nucl. Phys. A139, 713 (1968) for correct Figure 2.

[Berger-05] J. Berger et al., XCOM: Photon Cross Section Database (version 1.3). http://physics.nist.gov/xcom. National Institute of Standards and Technology, Gaithersburg, MD U.S.A. (2005).

[Bertozzi-05] William Bertozzi and Robert J. Ledoux, "Nuclear resonance fluorescence imaging in nonintrusive cargo inspection,” Nuc. Ins. Meth. Phys. Res. B 241 (2005) 820-825.

[Bertozzi-08] W. Bertozzi et al., "Nuclear Resonance Fluorescence Excitations near $2 \mathrm{MeV}$ in ${ }^{235} \mathrm{U}$ and

${ }^{239} \mathrm{Pu}$ " Physical Review C 78, 041601 (R) (2008)

[Firestone-99] Richard B. Firestone, Table of Isotopes, 8th ed. vol II. New York: John Wiley \& Sons, 1999, pp. 2759-2764.

[Kniessl-96] U Kniessl, HH Pitz and A Zilges, "Investigation of Nuclear Structure by Resonance Fluorescence Scattering,” Prog. Part. Nucl. Phys. 37, 349 (1996).

[Metzger-59] Franz R. Metzger, “Resonance Fluorescence in Nuclei,” Prog. Nucl Phys. 7, 54-88 (1959).

[Rullhusen-82] P. Rullhusen, U. Zurmühl, W. Mückenheim, F. Smend, and M. Schumacher, Nucl. Phys. A 382, 79 (1982).

[Tuli-87] J. Tuli. “Evaluated Nuclear Structure Data File,” BNL-NCS-51655- Rev 87, 198 (1987). 



\section{Distribution}

No. of

$\underline{\text { Copies }}$

1 Bob Mayo

DOE, NA-22

1 Bob Runkle

DOE, NA-22
No. of

\section{$\underline{\text { Copies }}$}

3 Internal Distribution
Pacific Northwest National Laboratory
$\begin{aligned} & \text { P.O. Box } 999 \\ & \text { Richland, WA } 99352 \\ & \text { Glen Warren } \\ & \text { Jac Caggiano } \\ & \text { Karl Pitts }\end{aligned} \quad$ P8-25

Distr.1 


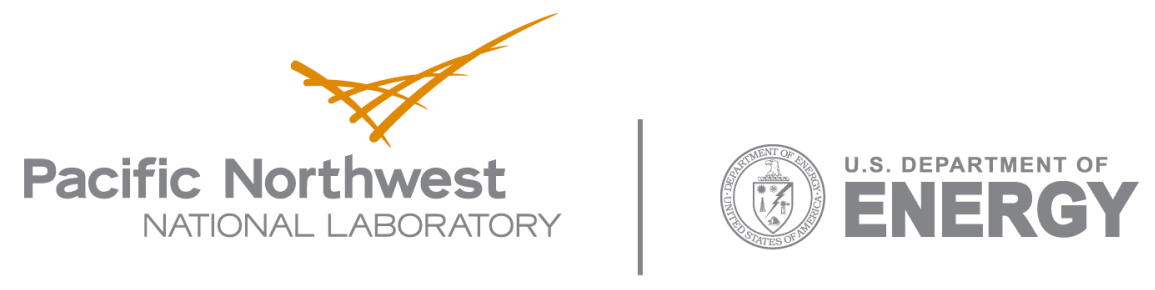

902 Battelle Boulevard

P.O. Box 999

Richland, WA 99352

1-888-375-PNNL (7665)

www.pnl.gov 